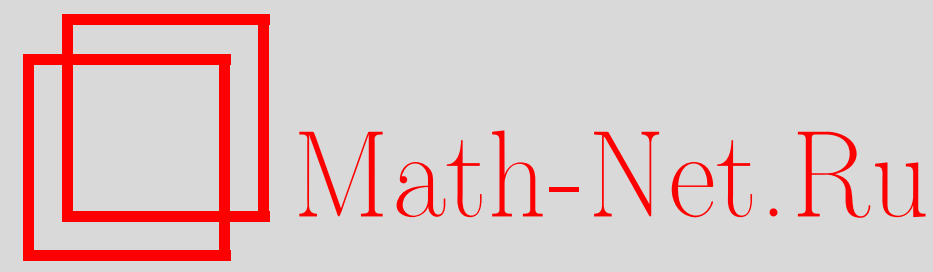

В. С. Рабинович, Алгебры сингулярных интегральных операторов на сложных контурах с узлами, являющимися точками логарифмического завихрения, Изв. РАН. Сер. матем., 1996, том 60, выпуск 6, 169-200

DOI: https://doi.org/10.4213/im99

Использование Общероссийского математического портала Math-Net.Ru подразумевает, что вы прочитали и согласны с пользовательским соглашением

http://www . mathnet.ru/rus/agreement

Параметры загрузки:

IP : 44.207 .124 .84

26 апреля 2023 г., 07:53:59 
УДК 517.95

\author{
В. С. Рабинович
}

\title{
Алгебры сингулярных интегральных операторов на сложных контурах с узлами, являющимися точками логарифмического завихрения
}

\begin{abstract}
Рассматривается банахова алгебра $\mathfrak{M}_{p}(\Gamma, \omega)$, порожденная сингулярными интегральными операторами, действующими в пространствах $L_{p}(\Gamma, \omega)$, где $\omega-$ степенной вес, $\Gamma$ - составной контур с узлами, являющимися точками завихрения логарифмического или более слабого характера, и операторами умножения на ограниченные функции, допускающие разрывы второго рода. Описана алгебра символов, и в терминах символов даны условия фредгольмовости операторов из алгебры $\mathfrak{M}_{p}(\Gamma, \omega)$. Существенную роль в работе играют теоремы о локальной обратимости псевдодифференциальных операторов и оценки их локальных норм.
\end{abstract}

Библиографияя: 23 наименования.

\section{Введение}

K настоящему времени теория банаховых алгебр, порожденных сингулярными интегральными операторами (СИО) на сложных кусочно-ляпуновских контурах с коэффициентами, имеющими разрывы первого рода, в основном построена (см., например, монографии [1]-[5] и статьи [6]-[8]). Отметим работу [9], посвященную СИО на сложных контурах с каспами, а также работу [10], в которой исследовались СИО со сдвигом на сложных контурах.

Основным аппаратом исследования операторов из указанных выше классов является локальный принцип в одной из его форм и анализ локальной алгебры, порожденной операторами умножения на кусочно-постоянные функции и СИО на системе лучей, выходяших из одной точки. При этом вычисление локальных представителей с точностью до замены переменных проводилось методом "замораживания", а сами локальные представители являлись операторами меллиновской свертки.

Алгебры, порожденные СИО на кривых Карлесона и операторами умножения на ограниченные функции, имеюшие разрывы второго рода, изучены только в некоторых частных ситуациях. Банаховы алгебры, порожденные операторами Теплища и Ханкеля на прямой или окружности и операторами умножения на кусочно-квазинепрерывные функции, рассматривались в работах [11]-[14]. В работе [15] рассматривалась задача Римана на негладких открытых кривых с точками логарифмического завихрения, а в работе [16] изучена банахова алгебра операторов, порожденная СИО и операторами умножения на кусочно-непрерывные функции, действующими в пространствах $L_{p}(\Gamma, \omega), 1<p<\infty$, где $\omega$ - вес Макенхаупта,

Работа выполнена при поддержке Российского фонда фундаментальных исследований, грант № 96-01-01195.

(C) В.С. РАвинович 1996 
$\Gamma$ - спрямляемый замкнутый контур, имеющий точки завихрения логарифмического характера.

В настоящей статье рассматриваются алгебры операторов $\mathfrak{M}_{p}(\Gamma, \omega)$, действующих в пространствах $L_{p}(\Gamma, \omega)$ со степенными весами $\omega$, порожденные СИО на сложных контурах Г с узлами, являющимися точками логарифмического завихрения более общего характера, чем в работе [16], и операторами умножения на функции, имеющие разрывы второго рода, из класса, являющегося замыканием по sup-норме на Г множества непрерывно дифференцируемых на $\Gamma / F(F$ - множество узлов) функций, удовлетворяющих условию

$$
\lim _{\Gamma \in x \rightarrow z}|x-z| a^{\prime}(z)=0, \quad z \in F .
$$

Кривые $\left\{\gamma_{j}\right\}_{j=1}^{N}$, составляющие контур в окрестности узла $z$, имеют параметризацию:

$$
\begin{aligned}
\gamma_{j} & =\left\{x \in \mathbb{C}: \quad x=z+\varphi_{j}(s), \quad \varphi_{j}(s)=s \exp i \omega_{j}(s), \quad s \in\left[0, s_{j}\right]\right\}, \\
\text { где } \omega_{j}(s) & =\delta \ln s+\theta_{j}(s), \delta \in \mathbb{R}, \theta_{j}(s) \in C^{1}\left(\left(0, s_{j}\right]\right), \lim _{s \rightarrow+0} s \theta_{j}^{\prime}(s)=0 .
\end{aligned}
$$

Продвижение в расширении класса контуров и кривых, для которых удается построить законченную теорию фредгольмовости, стало возможным благодаря привлечению для локального анализа СИО из указанных выше классов техники псевдодифференциальных операторов (ПДО). Сочетание локального принципа и техники ПДО позволяет построить символическое исчисление и дать условия фредгольмовости операторов из алгебры $\mathfrak{M}_{p}(\Gamma, \omega)$.

Отметим, что впервые техника ПДО для исследования СИО на контурах с точками завихрения была предложена в заметке автора [17], где рассматривался случай $p=2$, а кривые, составляющие узел, имели параметризацию $(*)$, в которой $\delta=0$.

Данная работа состоит из четырех параграфов. В $§ 1$ рассмотрены ПДО, действуюшие в $L_{p}(\mathbb{R}), 1<p<\infty$, с медленно меняющимися на бесконечности символами. Даны критерии локальной обратимости в бесконечно удаленных точках и фредпольмовости, а также приведены оценки локальных норм; $\S 2$ посвящен переносу этих результатов на ПДО Меллина. (Результаты этих параграфов, на наш взгляд, имеют самостоятельное значение и могут быть использованы во многих ситуациях.) В $\S 3$ строятся и исследуются банаховы алгебры, порожденные СИО и операторами умножения на функции, имеющие разрывы второго рода. В $\S 4$ рассмотрен случай замкнутого контура с точками завихрения и дана формула индекса, доказательство которой основано на формуле Федосова (см. [18], [19]) для ПДО на $\mathbb{R}$.

\section{§ 1. Псевдодифференциальные операторы на $\mathbb{R}$ нулевого порядка}

1. Псевдодифференциальные операторы. Будем использовать следующие обозначения: если $\mathfrak{B}$ - комплексное банахово пространство, то $\mathfrak{L}(\mathfrak{B})$ - пространство линейных ограниченных операторов, действующих в $\mathfrak{B}, \mathfrak{K}(\mathfrak{B})$ - двусторонний идеал компактных операторов в $\mathfrak{B}$. Через $L_{p}^{N}=L_{p}\left(\mathbb{R}, \mathbb{C}^{N}\right), p \in(1, \infty)$, обозначим банахово пространство измеримых на $\mathbb{R}$ вектор-функций с нормой

$$
\|u\|_{L_{p}^{N}}=\left(\int_{\mathbb{R}} \sum_{j=1}^{N}\left|u_{j}(x)\right|^{p} d x\right)^{1 / p} .
$$


ОПРЕДЕЛЕниЕ 1.1. Через $S_{1,0}^{m}\left(\mathbb{R}^{2}\right)$ обозначим класс таких $C^{\infty}$-функций на $\mathbb{R}^{2}$, что для любых $\alpha, \beta$ найдутся константы $C_{\alpha, \beta}>0$ такие, что выполнены неравенства

$$
\left|\partial_{x}^{\beta} \partial_{\xi}^{\alpha} a(x, \xi)\right| \leqslant C_{\alpha, \beta}\langle\xi\rangle^{m-\alpha}, \quad\langle\xi\rangle=\left(1+\xi^{2}\right)^{1 / 2} .
$$

Через $S_{1,0}^{m}\left(\mathbb{R}^{2}, \mathbb{C}^{N \times N}\right)$ обозначим класс матрищ-функций

$$
a(x, \xi)=\left(a_{i j}(x, \xi)\right)_{i, j=1}^{n}, \quad a_{i j}(x, \xi) \in S_{1,0}^{m}\left(\mathbb{R}^{2}\right) .
$$

Матрице-функции $a(x, \xi) \in S_{1,0}^{m}\left(\mathbb{R}^{2}, \mathbb{C}^{N \times N}\right)$, называемой символом, сопоставим ПДО $A=a(x, D)$ по формуле

$$
A u=a(x, D) u=(2 \pi)^{-1} \iint a(x, \xi) u(y) \exp (i(x-y) \xi) d y d \xi,
$$

где $u \in C_{0}^{\infty}\left(\mathbb{R}, \mathbb{C}^{N}\right) ;$ интегрирование в $(1.2)$ ведется по всему пространству $\mathbb{R}^{2}$. Интеграл в формуле (1.2) понимается либо как повторный, либо как осциллирующий [19], [20].

Через $O P S_{1,0}^{m}(N)$ обозначим класс ПДО с символами $a(x, \xi) \in S_{1,0}^{m}\left(\mathbb{R}^{2}, \mathbb{C}^{N \times N}\right)$.

ОПРЕДЕЛЕНИЕ 1.2. Пусть $a(x, y, \xi) \in C^{\infty}\left(\mathbb{R}^{3}, \mathbb{C}^{N \times N}\right)$ и вьполнены оценки

$$
\left|\partial_{x}^{\beta} \partial_{y}^{\gamma} \partial_{\xi}^{\alpha} a_{i j}(x, y, \xi)\right| \leqslant C_{\alpha, \beta, \gamma}\langle\xi\rangle^{m-\alpha}, \quad i, j=1, \ldots, N,
$$

для любых $\alpha, \beta, \gamma$. В этом случае будем говорить, что $a(x, y, \xi) \in S_{1,0}^{m}\left(\mathbb{R}^{3}, \mathbb{C}^{N \times N}\right)$.

Сопоставим $a(x, y, \xi) \in S_{1,0}^{m}\left(\mathbb{R}^{3}, \mathbb{C}^{N \times N}\right)$ оператор

$$
A u=(2 \pi)^{-1} \iint a(x, y, \xi) u(y) \exp (i(x-y) \xi) d y d \xi,
$$

где $u \in C_{0}^{\infty}\left(\mathbb{R}, \mathbb{C}^{N}\right)$, который называется ПДО с двойным символом. (В случае, когда не пишутся пределы интегрирования, это означает, что интегрирование ведется по $\mathbb{R}^{2}$.)

ПрЕДЛОЖЕНИЕ 1.2 [20]. (а) Eсли $A, B \in O P S_{1,0}^{0}(N)$, mo $A B \in O P S_{1,0}^{0}(N) u$ символ $\sigma_{A B}(x, \xi)$ оператора $A B$ вычисляется по формуле

$$
\sigma_{A B}(x, \xi)=(2 \pi)^{-n} \iint a(x, \xi+\eta) b(x+y, \xi) \exp (-i(y, \eta)) d y d \eta .
$$

(b) Eсли $A \in O P S_{1,0}^{0}(N)$, то формально сопряженный оператор $A^{*}$ принадлежит $O P S_{1,0}^{0}(N)$ u

$$
\sigma_{A^{*}}(x, \xi)=(2 \pi)^{-n} \iint a^{*}(x+y, \xi+\eta) \exp (-i(y, \xi)) d y d \xi .
$$

(c) Eсли а $(x, y, \xi) \in S_{1,0}^{0}\left(\mathbb{R}^{3}, \mathbb{C}^{N \times N}\right)$, то оператор (1.3) принадлежит OР $S_{1,0}^{0}(N)$ и его символ $\sigma_{A}(x, \xi)$ вичисляется по формуле

$$
\sigma_{A}(x, \xi)=(2 \pi)^{-1} \iint a(x, x+y, \xi+\eta) \exp (-i(y, \eta)) d y d \eta
$$


где интегралы в (1.4)-(1.6) понимаются как осциллирующие.

(d) Oператор $A \in O P S_{1,0}^{0}(N)$ ограничен в $L_{p}^{N}=L_{p}\left(\mathbb{R}, \mathbb{C}^{N}\right) u\|A\|_{\mathfrak{L}\left(L_{p}^{N}\right)} \leqslant M$, əдe

$$
M=\max _{1 \leqslant i, j \leqslant N} \max _{\alpha \leqslant 2, \beta \leqslant 1} \sup _{(x, \xi) \in \mathbb{R}^{2}}\left|\partial_{x}^{\beta} \partial_{\xi}^{\alpha} a_{i, j}(x, \xi)\right|\langle\xi\rangle^{\alpha}
$$

для операторов вида (1.2) и

$$
M=\max _{1 \leqslant i, j \leqslant N} \max _{\alpha \leqslant 2, \beta \leqslant 1, \gamma \leqslant 1} \sup _{(x, y, \xi) \in \mathbb{R}^{3}}\left\|\partial_{x}^{\beta} \partial_{y}^{\gamma} \partial_{\xi}^{\alpha} a_{i, j}(x, y, \xi)\right\|\langle\xi\rangle^{\alpha}
$$

для операторов вида (1.3).

ПРЕДЛОЖЕНИЕ 1.3 (см. [23]). Пусть $A \in O P S_{1,0}^{0}(N)$ и обратим в $L_{p}\left(\mathbb{R}, \mathbb{C}^{N}\right)$, $p \in(1, \infty) ;$ тогда $A^{-1} \in O P S_{1,0}^{0}(N)$.

\section{2. ПДО с медленно меняющимся при $x \rightarrow \infty$ символом.}

ОПРЕДЕЛЕНИЕ 1.3 . Пусть $a(x, \xi) \in S_{1,0}^{0}\left(\mathbb{R}^{2}, \mathbb{C}^{N \times N}\right)$. Будем говорить, что символ $a(x, \xi)$ медленно меняется при $x \rightarrow \infty$, если

$$
\lim _{x \rightarrow \infty} \sup _{\xi \in \mathbb{R}}\left|\partial_{x}^{\beta} \partial_{\xi}^{\alpha} a_{i j}(x, \xi)\right|\langle\xi\rangle^{\alpha}=0 \quad \forall \alpha, \forall \beta \neq 0 .
$$

Будем говорить, что двойной символ $а(x, y, \xi) \in S_{1,0}^{0}\left(\mathbb{R}^{3}, \mathbb{C}^{N \times N}\right)$ медленно меняется при $x, y \rightarrow \infty$, если

$$
\lim _{x \rightarrow \infty} \sup _{\xi \in \mathbb{R}^{n}, y \in K}\left|\partial_{x}^{\beta} \partial_{y}^{\gamma} \partial_{\xi}^{\alpha} a(x, x+y, \xi)\right|\langle\xi\rangle^{\alpha}=0 \quad \forall \alpha, \beta, \gamma: \quad \beta+\gamma \neq 0,
$$

где $K$ - произвольный компакт в $\mathbb{R}$.

Отметим, что класс ПДО с медленно меняющимися символами впервые был введен в работе [21] (см. также [22]).

ОПРЕДЕЛЕниЕ 1.4 . Обозначим через $\widetilde{S}_{1,0}\left(\mathbb{R}^{2}, \mathbb{C}^{N \times N}\right)$ класс медленно меняющихся при $x \rightarrow \infty$ символов, а класс символов, удовлетворяющих условиям

$$
\lim _{x \rightarrow \pm \infty} \sup _{\xi \in \mathbb{R}}\left|\partial_{x}^{\beta} \partial_{\xi}^{\alpha} a_{i j}(x, \xi)\right|\langle\xi\rangle^{\alpha}=0 \quad \forall \alpha, \forall \beta,
$$

обозначим через $\mathcal{T}_{1,0}^{ \pm}\left(\mathbb{R}^{2}, \mathbb{C}^{N \times N}\right)$.

Положим $\mathcal{T}_{1,0}\left(\mathbb{R}^{2}, \mathbb{C}^{N \times N}\right)=\mathcal{T}_{1,0}^{+}\left(\mathbb{R}^{2}, \mathbb{C}^{N \times N}\right) \cap \mathcal{T}_{1,0}^{-}\left(\mathbb{R}^{2}, \mathbb{C}^{N \times N}\right)$. Соответствуюшие классы ПДО обозначим $O P \widetilde{S}_{1,0}(N), O P \mathcal{T}_{1,0}^{ \pm}(N), O P \mathcal{T}_{1,0}(N)$.

ПРЕДЛОЖЕНИЕ 1.4. (а) Если $A, B \in O P \widetilde{S}_{1,0}(N)$, mo $A B \in O P \widetilde{S}_{1,0}(N) u$ символ $\sigma_{A B}(x, \xi)$ оператора $A B$ вычисляется по формуле

$$
\sigma_{A B}(x, \xi)=a(x, \xi) b(x, \xi)+t(x, \xi),
$$

əде $t(x, \xi) \in \mathcal{T}_{1,0}\left(\mathbb{R}^{2}, \mathbb{C}^{N \times N}\right) \cap S_{1,0}^{-1}\left(\mathbb{R}^{2}, \mathbb{C}^{N \times N}\right)$.

(b) Если $A \in O P \widetilde{S}_{1,0}(N)$, то формально сопряженный оператор $A^{*}$ принадлежит $O P \widetilde{S}_{1,0}(N)$ и его символ вычисляется по формуле

$$
\sigma_{A^{*}}(x, \xi)=a^{*}(x, \xi)+t(x, \xi),
$$


əде $t(x, \xi) \in \mathcal{T}_{1,0}\left(\mathbb{R}^{2}, \mathbb{C}^{N \times N}\right) \cap S_{1,0}^{-1}\left(\mathbb{R}^{2}, \mathbb{C}^{N \times N}\right)$.

(c) Eсли $а(x, y, \xi) \in \widetilde{S}_{1,0}\left(\mathbb{R}^{3}, \mathbb{C}^{N \times N}\right)$ и $A$ - соответствующий ПДО с двойным символом, то

$$
\sigma_{A}(x, \xi)=a(x, x, \xi)+t(x, \xi),
$$

әде $t(x, \xi) \in \mathcal{T}_{1,0}\left(\mathbb{R}^{2}, \mathbb{C}^{N \times N}\right) \cap S_{1,0}^{-1}\left(\mathbb{R}^{2}, \mathbb{C}^{N \times N}\right)$.

(d) Oператоры класса $O P \mathcal{T}_{1,0}(N) \cap O P S_{1,0}^{-1}(N)$ компактны в $L_{p}\left(\mathbb{R}, \mathbb{C}^{N}\right)$, $p \in(1, \infty)$.

Доказательство проводится точно так же, как и доказательство предложения 2.1 работы [22].

3. Алгебры $M_{p}(N), \mathscr{M}_{p}^{ \pm}(N), \mathscr{M}_{p}(N)$.

ОПРЕДЕЛЕНИЕ 1.5. Через $M_{p}(N)$ обозначается множество мультипликаторов в $L_{p}\left(\mathbb{R}, \mathbb{C}^{N}\right)$, т.е. таких измеримых на $\mathbb{R}$ матриц-функций, что оператор

$$
a(D) u=(2 \pi)^{-1} \int_{\mathbb{R}} a(\xi) \hat{u}(\xi) e^{i(x, \xi)} d \xi, \quad u \in C_{0}^{\infty}\left(\mathbb{R}, \mathbb{C}^{N}\right),
$$

где $\hat{u}(\xi)=\int_{\mathbb{R}} u(x) e^{-i(x, \xi)} d x$ - преобразование Фурье $u(x)$, продолжается до ограниченного оператора в $L_{p}\left(\mathbb{R}, \mathbb{C}^{N}\right)$.

Норма в $M_{p}(N)$ вводится по формуле $\|a\|_{M_{p}(N)}=\|a(D)\|_{\mathfrak{L}\left(L_{p}^{N}\right)}$.

Через $\mathscr{M}_{p}(N)$ обозначим банахову алгебру ограниченных, непрерывных на $\mathbb{R}$ матриц-функций $a(y, \cdot)$ со значениями в $M_{p}(N)$, нормой

$$
\|a\|_{\mathscr{M}_{p}(N)}=\sup _{y \in \mathbb{R}}\|a(y, \cdot)\|_{M_{p}(N)}
$$

и естественной операцией умножения матриц-функций.

Через $\mathscr{M}_{p}^{0 \pm}(N)$ обозначим подалгебру $\mathscr{M}_{p}(N)$, состояшую из таких матриц-функций $a \in \mathscr{M}_{p}(N)$, что

$$
\lim _{y \rightarrow \pm \infty}\|a(y, \cdot)\|_{M_{p}(N)}=0,
$$

а через $\mathscr{M}_{p}^{0}(N)$ обозначим $\mathscr{M}_{p}^{0+}(N) \cap \mathscr{M}_{p}^{0-}(N)$.

Будем обозначать через $\widetilde{\mathscr{M}}_{p}^{+}(N)=\mathscr{M}_{p}(N) / \mathscr{M}_{p}^{0+}(N)$ фактор-алгебру с естественной нормой

$$
\|\hat{a}\|_{\mathscr{M}_{p}^{+}(N)}=\lim _{R \rightarrow+\infty} \sup _{y \in(R,+\infty)}\|a(y, \cdot)\|_{M_{p}(N)},
$$

где $a \in \mathscr{M}_{p}(N)$ - произвольньй представитель факторкласса $\hat{a} \in \widetilde{\mathscr{M}}_{p}^{+}(N)$.

Аналогично вводятся факторалгебры

$$
\widetilde{\mathscr{M}}_{p}^{-}(N)=\mathscr{M}_{p}(N) / \mathscr{M}_{p}^{0-}(N) \text { и } \widetilde{\mathscr{M}}_{p}(N)=\mathscr{M}_{p}(N) / \mathscr{M}_{p}^{0}(N) .
$$

Отметим некоторые свойства банаховой алгебры $\widetilde{\mathscr{M}}_{p}^{+}(N)$. (Аналогичными свойствами обладают алгебры $\widetilde{\mathscr{M}}_{p}^{-}(N), \widetilde{\mathscr{M}}_{p}(N)$.) Э Это алгебра с единицей, $\widetilde{\mathscr{M}}_{p}^{+}(N)=\widetilde{\mathscr{M}}_{q}^{+}(N)\left(p^{-1}+q^{-1}=1,1<p<\infty\right), \widetilde{\mathscr{M}}_{p}^{+}(N) \subset \widetilde{\mathscr{M}}_{2}^{+}(N)$ и это вложение 
топологическое, причем алгебра $\widetilde{\mathscr{M}}_{2}^{+}(N)$ изометрически изоморфна факторалгебpe

$$
C_{b}\left(\mathbb{R}, L_{\infty}\left(\mathbb{R}, \mathbb{C}^{N \times N}\right)\right) / C_{0}^{+}\left(\mathbb{R}, L_{\infty}\left(\mathbb{R}, \mathbb{C}^{N \times N}\right)\right),
$$

где $C_{b}\left(\mathbb{R}, L_{\infty}\left(\mathbb{R}, \mathbb{C}^{N \times N}\right)\right)$ - замыкание алгебраического тензорного произведения $\left.C_{b}(\mathbb{R}) \otimes L_{\infty}\left(\mathbb{R}, \mathbb{C}^{N \times N}\right)\right)$ по норме

$$
\|a\|=\sup _{y \in \mathbb{R}}\|a(y, \cdot)\|_{L_{\infty}\left(\mathbb{C}^{N \times N}\right)},
$$

$C_{0}^{+}\left(\mathbb{R}, L_{\infty}\left(\mathbb{R}, \mathbb{C}^{N \times N}\right)\right)$ - двухсторонний идеал в $C_{b}\left(\mathbb{R}, L_{\infty}\left(\mathbb{R}, \mathbb{C}^{N \times N}\right)\right)$, состоящий из таких функций, что

$$
\lim _{y \rightarrow+\infty}\|a(y, \cdot)\|_{L_{\infty}\left(\mathbb{C}^{N \times N}\right)}=0 .
$$

Алгебра $\mathscr{M}_{p}(N)(1<p<\infty)$ является алгеброй с инволюцией: $a(y, \xi) \rightarrow$ $a^{*}(y, \xi)$, где $a^{*}(y, \xi)$ - символ оператора $a^{*}\left(y, D_{x}\right)$; следовательно, таковыми являются и алгебры $\widetilde{\mathscr{M}}_{p}^{+}(N), \widetilde{\mathscr{M}}_{p}^{-}(N), \widetilde{\mathscr{M}}_{p}(N)$.

Введенная таким образом инволюция обладает всеми свойствами, перечисленными в [5, с. 44]. Отсюда следует наполненность алгебры $\widetilde{\mathscr{M}}_{p}^{+}(N)$ в $\widetilde{\mathscr{M}}_{2}^{+}(N)$ при $1<p<\infty$, т.е. если элемент $a \in \widetilde{\mathscr{M}}_{p}^{+}(N)$ обратим в $\widetilde{\mathscr{M}}_{2}^{+}(N)$, то $a^{-1} \in \widetilde{\mathscr{M}}_{p}^{+}(N)$.

Таким образом, справедливо следующее

ПРЕДЛОЖЕНИЕ 1.5. Факторкласс $\hat{a} \in \widetilde{\mathscr{M}}_{p}^{+}(N), \quad 1<p<\infty$, обратим $в$ $\widetilde{\mathscr{M}}_{p}^{+}(N)$ тогда и только тогда, когда

$$
d_{+}(a)=\lim _{R \rightarrow+\infty} \inf _{(x, \xi) \in(R,+\infty) \times \mathbb{R}}|\operatorname{det} a(x, \xi)|>0,
$$

где а - произвольный представитель факторкласса а̂. (Аналогичным образом формулируется условие обратимости факторкласса $\hat{a} \in \widetilde{\mathscr{M}}_{p}^{-}(N)$.)

Необходимое и достаточное условия обратимости факторкласса $\hat{a} \in$ $\mathscr{M}_{p}(N)$ состоят в том, что

$$
d(a)=\lim _{R \rightarrow+\infty} \inf _{|x|>R, \xi \in \mathbb{R}}|\operatorname{det} a(x, \xi)|>0,
$$

где а - произвольный представитель факторкласса $\hat{a}$.

4. Локальная обратимость ПДО в бесконечно удаленной точке. Пусть функция $\varphi(x) \in C^{\infty}(\mathbb{R})$ равна 1 при $x \geqslant 2$ и равна 0 при $x \leqslant 1$, $0 \leqslant \varphi(x) \leqslant 1, \varphi_{R}(x)=\varphi(x / R)$.

ОПРЕДЕЛЕНИЕ 1.6. Будем говорить, что оператор $A \in \mathfrak{L}\left(L_{p}^{N}\right)$ локально обратим слева (справа) в точке $+\infty$, если найдутся такое число $R>0$ и такие операторы $B^{\prime}, B^{\prime \prime} \in \mathfrak{L}\left(L_{p}^{N}\right)$, что $B_{R}^{\prime} A \varphi_{R}=\varphi_{R}\left(\varphi_{R} A B_{R}^{\prime \prime}=\varphi_{R}\right)$.

Оператор называется локально обратимым в точке $+\infty$, если он локально обратим в этой точке слева и справа.

Аналогично определяется локальная обратимость в точках $-\infty,+\infty$.

Положим

$$
d_{+}(A)=\lim _{R \rightarrow+\infty} \inf _{(y, \xi) \in(R,+\infty) \times \mathbb{R},\|h\|=1}\left\|a\left(y, D_{x}\right) h\right\|_{L_{p}^{N}} .
$$


Теорема 1.1. Пусть $A \in O P S_{1,0}^{+}(N)$. Тогда следующие условия әквивалентни:

(a) $A: L_{p}\left(\mathbb{R}, \mathbb{C}^{N}\right) \rightarrow L_{p}\left(\mathbb{R}, \mathbb{C}^{N}\right)$ локально обратим в точке $+\infty$;

(b) $d_{+}($a) $>0$;

(c) существуют левый и правый локально обратные в точке $+\infty$ операторы, принадлежащие классу ОР $\widetilde{S}_{1,0}(N)$.

ЗАмечАниЕ. Как следует из предложения 1.5, условие (b) равносильно услоВИю

$$
\lim _{R \rightarrow+\infty} \inf _{(x, \xi) \in(R,+\infty) \times \mathbb{R}}|\operatorname{det} a(x, \xi)|>0 .
$$

ДокаЗАТЕЛЬСтво. (a) $\Rightarrow(\mathrm{b})$. Из локальной обратимости оператора $A$ в точке $+\infty$ следует сушествование таких чисел $R_{0}>0, C>0$, что для всех $R>R_{0}$

$$
\left\|A \varphi_{R} u\right\|_{L_{p}^{N}} \geqslant C\left\|\varphi_{R} u\right\|_{L_{p}^{N}} \quad \forall u \in C_{0}^{\infty}\left(\mathbb{R}, \mathbb{C}^{N}\right) .
$$

Пусть последовательность $y_{n} \rightarrow+\infty$; тогда для достаточно больших номеров $n>n_{0} \varphi_{R}\left(\tau_{y_{n}} u\right)=\tau_{y_{n}} u\left(\right.$ где $\left(\tau_{h} u\right)(x)=u(x-h)$ - оператор сдвига). Таким образом, из (1.12) и изометричности в $L_{p}^{N}$ оператора сдвига следует, что

$$
\left\|\tau_{-y_{n}} A \tau_{y_{n}} u\right\|_{L_{p}^{N}} \geqslant C\|u\|_{L_{p}^{N}} \quad \forall u \in C_{0}^{\infty}\left(\mathbb{R}, \mathbb{C}^{N}\right) .
$$

Заметим, что $\tau_{-y_{n}} A \tau_{y_{n}}=a\left(x+y_{n}, D_{x}\right)$. Используя то, что $a(x, D) \in$ $O P \widetilde{S}_{1,0}(N)$, и предложение $1.2,(\mathrm{~b})$, нетрудно показать, что последовательность операторов

$$
q_{n}(x, D)=a\left(x+y_{n}, D_{x}\right)-a\left(y_{n}, D_{x}\right)
$$

сильно сходится к нулю в $L_{p}^{N}$. Таким образом, из формулы (1.13) следует, что для любой последовательности $y_{n} \rightarrow+\infty$

$$
\underline{\lim }_{n \rightarrow \infty}\left\|a\left(y_{n}, D_{x}\right) u\right\|_{L_{p}^{N}} \geqslant C\|u\|_{L_{p}^{N}} \quad \forall u \in C_{0}^{\infty}\left(\mathbb{R}, \mathbb{C}^{N}\right) .
$$

Из формулы (1.14) находим, что

$$
\lim _{R \rightarrow \infty} \inf _{y>R}\left\|a\left(y, D_{x}\right) u\right\|_{L_{p}^{N}} \geqslant C\|u\|_{L_{p}^{N}} \quad \forall u \in C_{0}^{\infty}\left(\mathbb{R}, \mathbb{C}^{N}\right)
$$

т.e. $d_{+}(A)>0$.

(b) $\Rightarrow$ (c). Пусть выполнено условие (b), а следовательно, условие (1.11), из которого следует существование такого числа $R_{0}>0$, что для $\forall R\left(>R_{0}\right)$ существует $a^{-1}(x, \xi), x>R$. Положим

$$
r_{R}(x, \xi)=\varphi_{R}(x) a^{-1}(x, \xi) \quad\left(\in \widetilde{S}_{1,0}^{+}\left(\mathbb{R}^{2}, \mathbb{C}^{N \times N}\right)\right), \quad R>R_{0} .
$$

Тогда

$$
r_{R}(x, D) a(x, D)=\varphi_{R}(x)+t(x, D),
$$

где $t(x, D) \in O P \mathcal{T}_{1,0}^{+}(N)$ в силу предложения $1.4,($ a). 
Выберем теперь $R^{\prime}>R$ настолько большим, чтобы $\varphi_{R}(x) \varphi_{R^{\prime}}(x)=\varphi_{R^{\prime}}(x)$. Тогда

$$
r_{R}(x, D) a(x, D)=\left(I+t(x, D) \varphi_{R}(x)\right) \varphi_{R^{\prime}}(x) .
$$

Так как $t(x, D) \in O P \mathcal{T}_{1,0}^{+}(N)$, то из предложения 1.2 ,(b) следует существование такого $R>R_{0}$, что $\left\|t(x, D) \varphi_{R}(x)\right\|_{\mathfrak{L}\left(L_{p}^{N}\right)}<1$. Оператор $\left(I+t(x, D) \varphi_{R}(x)\right)$ обратим в $L_{p}^{N}$ для достаточно большого $R$ и $\left(I+t(x, D) \varphi_{R}(x)\right)^{-1} \in O P S_{1,0}^{0}(N)$ в силу предложения 1.3 .

Таким образом, $A$ локально обратим слева в точке $+\infty$, и левый локально обратньй оператор $B_{l}=\left(I+t(x, D) \varphi_{R}(x)\right)^{-1} r_{R}(x, D) \in O P \widetilde{S}_{1,0}(N)$.

Теорема, аналогичная теореме 1.1 , дает условия локальной обратимости и в точках $-\infty,+\infty$.

5. Фредгольмовость и индекс ПДО класса $O P \widetilde{S}_{1,0}(N)$.

TeOPEMA 1.2. Onepamop $A=a(x, D) \in O P \widetilde{S}_{1,0}(N)$, действующий $в$ $L_{p}\left(\mathbb{R}, \mathbb{C}^{N}\right), 1<p<\infty$, фредгольмов тогда и только тогда, когда

$$
\lim _{R \rightarrow+\infty} \inf _{|x|+|\xi| \geqslant R}|\operatorname{det} a(x, \xi)|>0 .
$$

Если условие (1.16) выполнено, то индекс оператора А вычисляется по формуле

$$
\text { ind } A=-(2 \pi)^{-1}[\arg \operatorname{det} a(x, \xi)]_{\Gamma_{R^{\prime}, R^{\prime \prime}}},
$$

где приращение аргумента [·] берется вдоль контура

$$
\Gamma_{R^{\prime}, R^{\prime \prime}}=\partial \Pi\left(R^{\prime}, R^{\prime \prime}\right), \quad \Pi\left(R^{\prime}, R^{\prime \prime}\right)=\left\{(x, \xi) \in \mathbb{R}^{2}:|x| \leqslant R^{\prime}, \quad|\xi| \leqslant R^{\prime \prime}\right\},
$$

причем контур $\Gamma_{R^{\prime}, R^{\prime \prime}}$ ориентирован положстельным образом, т.е. при его обходе против часовой стрелки $\Pi\left(R^{\prime}, R^{\prime \prime}\right)$ остается слева; числа $R^{\prime}, R^{\prime \prime}$ таковы, ито при $(x, \xi) \in \mathbb{R}^{2} \backslash \Pi\left(R^{\prime}, R^{\prime \prime}\right)$ существует $a^{-1}(x, \xi)$.

ДокАЗАТЕЛЬСтво. Необходимость и достаточность условия фредгольмовости (1.16) доказывается так же, как доказываются в теореме 1.1 необходимые и достаточные условия локальной обратимости. Заметим, что при $p=2$ это условие получено в [21].

Докажем формулу индекса (1.17). Пусть компоненты $a_{i j}(x, \xi)$ символа $a(x, \xi)$ удовлетворяют оценкам

$$
\left|\partial_{x}^{\beta} \partial_{\xi}^{\alpha} a_{i j}(x, \xi)\right| \leqslant C_{\alpha, \beta}\langle\xi\rangle^{-\alpha}\langle x\rangle^{-\beta}
$$

для любых мультииндексов $\alpha, \beta ; i, j=1, \ldots, N$. В этом случае для вычисления индекса ПДО применима формула Федосова [18], [19], согласно которой

$$
\text { ind } A=-(2 \pi i)^{-1} \int_{\Gamma_{R^{\prime}, R^{\prime \prime}}} \operatorname{Sp}\left(a^{-1} d a\right)=-(2 \pi)^{-1}[\arg \operatorname{det} a(x, \xi)]_{\Gamma_{R^{\prime}, R^{\prime \prime}}} .
$$

Формула (1.19) получена в [19] для операторов, действующих в $L_{2}^{N}$, однако, поскольку регуляризатор оператора $a(x, D)$ один и тот же во всех пространствах 
$L_{p}^{N}, 1<p<\infty$, эта формула справедлива и для оператора $a(x, D)$, действующего в $L_{p}^{N}$.

Нетрудно показать, используя предложение $1.2,(\mathrm{~d})$, что любой оператор $A \in$ $O P \widetilde{S}_{1,0}(N)$ можно аппроксимировать в $\mathfrak{L}\left(L_{p}^{N}\right)$ последовательностью $A_{n}$ ПДО с символами, удовлетворяющими оценкам (1.18). Переход к пределу в (1.19) позволяет заключить, что эта формула верна для ПДО класса $O P \widetilde{S}_{1,0}(N)$.

Пусть $\chi_{r}^{+}(x)$ - характеристическая функция луча $\mathbb{L}_{r}=\{x \in \mathbb{R}: x>r\}$, $\chi_{r}^{-}(x)=1-\chi_{r}^{+}(x)$.

В дальнейшем полезным является следуюшее

ПРЕДЛОЖЕНИЕ 1.6. Пусть $a(x, D) \in O P \widetilde{S}_{1,0}(N)$ и его символ $a(x, \xi)$ тождественно равен 1 при $x \leqslant r+\varepsilon(\varepsilon>0)$.

Oператор $\chi_{r}^{+} a(x, D) \chi_{r}^{+}$фредгольмов в $L_{p}\left(\mathbb{L}_{r}, \mathbb{C}^{N}\right)$ тогда и только тогда, когда оператор а $(x, D)$ фредгольмов в $L_{p}\left(\mathbb{R}, \mathbb{C}^{N}\right)$, причем индексы этих операторов совпадают.

ДокАЗАТЕЛЬСтво. Оператор $\chi_{r}^{+} a(x, D) \chi_{r}^{+}$фредгольмов в $L_{p}\left(\mathbb{T}_{r}, \mathbb{C}^{N}\right)$ тогда и только тогда, когда фредгольмов в $L_{p}\left(\mathbb{R}, \mathbb{C}^{N}\right)$ оператор $A_{1}=\chi_{r}^{+} a(x, D) \chi_{r}^{+}+$ $\chi_{r}^{-}(x)$. Покажем, что $\tilde{a}(x, D)-A_{1}$ есть компактньй оператор. Действительно,

$$
\tilde{a}(x, D)-A_{1}=\chi_{r}^{+} \tilde{a}(x, D) \chi_{r}^{-}+\chi_{r}^{-} \tilde{a}(x, D) \chi_{r}^{+}+\chi_{r}^{-} \tilde{a}(x, D) \chi_{r}^{-}-\chi_{r}^{-} .
$$

Очевидно, что $\chi_{r}^{-} \tilde{a}(x, D) \chi_{r}^{-}=\chi_{r}^{-} ;$следовательно, из (1.20) следует, что

$$
\tilde{a}(x, D)-A_{1}=\chi_{r}^{+} \tilde{a}(x, D) \chi_{r}^{-}=\chi_{r}^{+}(\tilde{a}(x, D)-I) \chi_{r}^{-}
$$

Оператор $\tilde{a}(x, D)-I$ имеет символ, тождественно равный нулю на множестве $(-\infty, r+\varepsilon)$, поэтому

$$
\chi_{r}^{+}(\tilde{a}(x, D)-I) \chi_{r}^{-}=\psi(\tilde{a}(x, D)-I) \chi_{r}^{-}
$$

где $\psi$ - некоторая функция, принадлежащая $C^{\infty}(\mathbb{R}), \operatorname{supp} \psi \subset \mathbb{L}_{r}, \psi(x)=1$ при $x \geqslant r+\varepsilon$.

Из предложения 1.4 следует, что коммутатор $[\psi, \tilde{a}(x, D)]$ компактен в $L_{p}\left(\mathbb{R}, \mathbb{C}^{N}\right)$, $p \in(1, \infty)$, и, следовательно, оператор $\chi_{r}^{+} \tilde{a}(x, D) \chi_{r}^{-}$также компактен.

6. Оценки локальной и существенной норм оператора $A \in O P \widetilde{S}_{1,0}$.

ОПРЕДЕЛЕНИЕ 1.7. Пусть $A \in O P \widetilde{S}_{1,0}(N)$; тогда локальной нормой оператора $A$ в точке $\pm \infty$ назовем число

$$
\|A\|\left\|_{p, \pm}=\inf _{T \in O P \mathcal{T}_{1,0}^{ \pm}}\right\| A-T \|_{\mathfrak{L}\left(L_{p}^{N}\right)}
$$

Существенной нормой оператора $A \in \mathfrak{L}\left(L_{p}^{N}\right)$ называют число

$$
\|A\|\left\|_{p}=\inf _{T \in \mathfrak{K}\left(L_{p}^{N}\right)}\right\| A-T \|_{\mathfrak{L}\left(L_{p}^{N}\right)} .
$$


TeOpema 1.3. Пусть $A \in O P \widetilde{S}_{1,0}(N), \quad 1<p<\infty ;$ mozдa

$$
\|\hat{a}\|_{\widetilde{\mathscr{M}}_{p}^{ \pm}} \leqslant \mid\|A\|\left\|_{p, \pm} \leqslant C\right\| \hat{a} \|_{\widetilde{\mathscr{M}}_{p}^{ \pm}}
$$

с константой $C>0$, не зависящей от $A, p$.

Teоpema $1.3^{\prime}$. Пусть $A=a(x, \xi) \in O P \widetilde{S}_{1,0}(N), 1<p<\infty$. Положим

$$
\lim _{r \rightarrow+\infty} \sup _{|\xi| \geqslant r, x \in \mathbb{R}}\|a(x, \xi)\|_{p}=a_{\infty},
$$

әде $\|\cdot\|_{p}-$ норма $(N \times N)$-матрицы $a(x, \xi)$, индуцированная $l_{p}$-нормой $\mathbb{C}^{N}$. Положим

$$
|| \hat{a}||_{p}=\max \left\{\|\hat{a}\|_{\widetilde{\mathscr{M}}_{p}}, a_{\infty}\right\} .
$$

Справедлива следующая двухсторонняя оченка:

$$
\left\|\hat{a}||_{p} \leqslant\left.|||A|\right|_{p} \leqslant C \mid\right\| \hat{a} \|_{p_{p}} .
$$

Отметим, что ||$\hat{a} \mid \|_{p}$ есть норма в факторалгебре $\mathscr{M}_{p} /\left(\mathscr{M}_{p}^{0} \cap \mathscr{N}_{p}^{0}\right)$, где $\mathscr{N}_{p}^{0}-$ идеал в $\mathscr{M}_{p}$, состоящий из таких матриц-функций $a(x, \xi)$, что

$$
\lim _{r \rightarrow+\infty} \sup _{|\xi| \geqslant r, x \in \mathbb{R}}\|a(x, \xi)\|_{p}=0 .
$$

Проведем доказательство теоремы 1.3 только для знака + .

ДокАЗАТЕЛЬСТво. Докажем сначала оценку снизу в (1.21). Зададимся $\varepsilon>0$ и найдем оператор $T_{0} \in O P \mathcal{T}_{1,0}^{+}$такой, что

$$
\left\||| A||_{p,+} \geqslant\right\| A-T_{0}\|-\varepsilon \geqslant\|\left(A-T_{0}\right) u\left\|-\varepsilon \quad \forall u \in C_{0}^{\infty}(\mathbb{R}), \quad\right\| u \|_{L_{p}}=1 .
$$

Заменим в (1.18) функцию $u$ на $\tau_{y_{m}} u$, где $y_{m} \rightarrow+\infty$; тогда в силу изометричности оператора сдвига в $L_{p}^{N}$ имеем

$$
\left\|\left(A-T_{0}\right) \tau_{y_{m}} u\right\|=\left\|\tau_{-y_{m}}\left(A-T_{0}\right) \tau_{y_{m}} u\right\| .
$$

Операторные последовательности $Q_{m}=\tau_{-y_{m}} A \tau_{y_{m}}-a\left(y_{m}, D_{x}\right), \tau_{-y_{m}} T_{0} \tau_{y_{m}}$ сильно сходятся к нулю в $L_{p}^{N}$ при $m \rightarrow \infty$. Таким образом, из (1.22) и (1.23) следует, что

$$
\||| A \mid\|_{p,+} \geqslant \varlimsup_{m \rightarrow+\infty}\left\|a\left(y_{m}, D_{x}\right) u\right\|_{L_{p}^{N}}
$$

для любой функции $u \in C_{0}^{\infty}\left(\mathbb{R}, \mathbb{C}^{N}\right),\|u\|_{L_{p}^{N}}=1$, и любой последовательности $y_{m} \rightarrow+\infty$. Из (1.24) в силу произвольности $u \in C_{0}^{\infty}\left(\mathbb{R}, \mathbb{C}^{N}\right),\|u\|_{L_{p}^{N}}=1$, следует, что

$$
\||| A \mid\|_{p,+} \geqslant \varlimsup_{m \rightarrow+\infty}\left\|a\left(y_{m}, D_{x}\right)\right\|_{L_{p}^{N}},
$$

откуда и вытекает оценка снизу в (1.21).

Для доказательства оценки сверху в (1.21) нам потребуется приведенная ниже лемма. Для ее формулировки введем следуюшие обозначения.

Пусть $\alpha \in \mathbb{Z}$; положим $V_{\alpha}^{k}=\{x \in \mathbb{R}:|x-\alpha|<k\}$. Интервалы $V_{\alpha}^{k}$, $\alpha \in \mathbb{Z}$, образуют открытое покрытие $\mathbb{R}$ кратности $k+1$. Пусть $\varphi^{k}(x) \in C_{0}^{\infty}(\mathbb{R})$, $\operatorname{supp} \varphi^{k}(x) \in V_{0}^{k}, 0 \leqslant \varphi^{k}(x) \leqslant 1, \varphi_{\alpha}^{k}=\varphi(x-\alpha), \varphi^{k}(x) \varphi^{k+1}(x)=\varphi^{k}(x)$ и $\sum_{\alpha \in \mathbb{Z}} \varphi_{\alpha}^{k}(x)=1$. Положим $\varphi_{\alpha, R}^{k}(x)=\varphi_{\alpha}^{k}(x / R)$. 
ЛЕмма 1.1 [5]. Пусть $A_{\alpha}, a \in \mathbb{Z},-$ равномерно ограниченное семейство операторов, действующих в $L_{p}^{N}, \quad 1<p<\infty$. Тогда ряд $\sum_{\alpha \in \mathbb{Z}} \varphi_{\alpha, R}^{k}(x) A_{\alpha} \times$ $\varphi_{\alpha, R}^{r}(x)$ сильно сходится в $L_{p}^{N} u$

$$
\left\|\sum_{\alpha \in \mathbb{Z}} \varphi_{\alpha, R}^{k}(x) A_{\alpha} \varphi_{\alpha, R}^{r}(x)\right\|_{\mathcal{L}\left(L_{p}^{N}\right)} \leqslant(k+1)(r+1) \sup \left\|A_{\alpha}\right\|_{\mathcal{L}\left(L_{p}^{N}\right)} .
$$

Проведем доказательство оценки сверху в (1.21). Для заданного числа $\varepsilon>0$ найдется такое $R_{0}>0$, что

$$
\sup _{y \in(R,+\infty)}\left\|a\left(y, D_{x}\right)\right\|_{\mathfrak{L}\left(L_{p}^{N}\right)} \leqslant\|\hat{a}\|_{\widetilde{M}_{p}^{+}}+\varepsilon \quad \forall R>R_{0} .
$$

Оператор $A$ представим в виде

$$
\begin{aligned}
A=\sum_{\alpha \in \mathbb{Z}} \sum_{\beta \in \mathbb{Z}} \varphi_{\alpha, R}^{1}(x) A \varphi_{\beta, R}^{1}(x)= & \sum_{|\alpha-\beta| \leqslant L} \varphi_{\alpha, R}^{1}(x) A \varphi_{\beta, R}^{1}(x) \\
& +\sum_{|\alpha-\beta|>L} \varphi_{\alpha, R}^{1}(x) A \varphi_{\beta, R}^{1}(x) .
\end{aligned}
$$

Оценим второе слагаемое в правой части (1.26) (обозначим его через $T(L, R)$ ):

$$
T(L, R)=\sum_{|\alpha-\beta|>L} \varphi_{\alpha, R}^{1}(x) \varphi_{\alpha, R}^{2}(x) A \varphi_{\beta, R}^{2}(x) \varphi_{\beta, R}^{1}(x) .
$$

Выберем $L$ так, чтобы $\operatorname{supp} \varphi_{\alpha, R}^{2}(x) \cap \operatorname{supp} \varphi_{\beta, R}^{2}(x)=\varnothing$ при $|\alpha-\beta|>L$. Тогда, используя предложение $1.2,(\mathrm{a})$, нетрудно показать, что

$$
\left\|\varphi_{\alpha, R}^{2}(x) A \varphi_{\beta, R}^{2}(x)\right\|_{\mathfrak{L}\left(L_{p}^{N}\right)} \leqslant C R^{-1}
$$

где константа $C>0$ не зависит от $\alpha, \beta, R$.

Из леммы 1.1 следует, что

$$
\|T(R)\|_{\mathcal{L}\left(L_{p}^{N}\right)} \leqslant C R^{-1}
$$

с константой $C>0$, не зависяшей от $\alpha, \beta, R$.

Из условия, что $A \in O P \widetilde{S}_{1,0}^{+}$, и предложения $1.2,(\mathrm{~d})$ следует, что

$$
\lim _{\alpha \rightarrow+\infty}\left\|\varphi_{\alpha, R}^{2}(x)\left(a\left(x, D_{x}\right)-a\left(\alpha, D_{x}\right)\right) \varphi_{\beta, R}^{2}(x)\right\|_{\mathcal{L}\left(L_{p}^{N}\right)}=0
$$

для любого $R>0$.

Таким образом, из (1.29) и (1.30) следует, что $\forall \varepsilon>0$ найдутся числа $R^{\prime}>0$ и $m^{\prime} \in \mathbb{N}$ такие, что $\forall R>R^{\prime}, \forall m>m^{\prime}$

$$
\|A\|_{\mathfrak{L}\left(L_{p}^{N}\right)} \leqslant \sum_{|\alpha-\beta| \leqslant L, \alpha \leqslant m}\left\|\varphi_{\alpha, R}^{1}(x) A \varphi_{\beta, R}^{1}(x)\right\|+C \sup _{\alpha>m}\left\|a\left(\alpha, D_{x}\right)\right\|_{\mathfrak{L}\left(L_{p}^{N}\right)}+2 \varepsilon .
$$


Пусть $\Psi_{N}(x) \in C^{\infty}(\mathbb{R})$ равна 1 при $x>2 N>0$ и равна 0 при $x<N$. Выберем $N$ настолько большим, чтобы $\varphi_{\alpha, R}^{1}(x) \Psi_{N}(x)=0$, когда $R>R^{\prime}, m>m^{\prime}$. Тогда из оценки (1.31) следует, что

$$
\begin{array}{r}
\|\| A\|\|_{p,+} \leqslant\left\|\Psi_{N}(x) A\right\|_{\mathcal{L}\left(L_{p}^{N}\right)} \leqslant C \sup _{\alpha>m}\left\|\Psi_{N}(\alpha) a\left(\alpha, D_{x}\right)\right\|_{\mathcal{L}_{\left(L_{p}^{N}\right)}}+2 \varepsilon \\
\leqslant C \sup _{y>m}\left\|\Psi_{N}(y) a\left(y, D_{x}\right)\right\|_{\mathcal{L}\left(L_{p}^{N}\right)}+2 \varepsilon \leqslant C\|\hat{a}\|_{\mathcal{M}_{p}^{+}}+3 \varepsilon .
\end{array}
$$

Число $m$ в оценке (1.32) можно выбрать так, чтобы $\Psi_{N}(y)=1$ при $y \geqslant m$ и

$$
\sup _{y>m}\left\|\Psi_{N}(y) a\left(y, D_{x}\right)\right\|_{\mathcal{L}\left(L_{p}^{N}\right)}<\|\hat{a}\|_{\mathcal{M}_{p}^{+}}+\varepsilon / C
$$

Из (1.32) и (1.33) следует, что $\|\mid A\|\left\|_{p,+} \leqslant C\right\| \hat{a} \|_{\mathscr{M}_{p}^{+}}+3 \varepsilon$. Ввиду произвольности $\varepsilon>0$ отсюда и следует оценка сверху в (1.21).

7. Банахова алгебра $\mathfrak{U}_{p}(N)$ и некоторые ее факторалгебры. Через $\mathfrak{U}_{p}(N)$ обозначим банахову алгебру операторов, действуюших в пространстве $L_{p}^{N}$, $p \in(1, \infty)$, полученную замыканием в $\mathfrak{L}\left(L_{p}^{N}\right)$ множества псевдодифференциальных операторов $a(x, D) \in O P \widetilde{S}(N)$. Через $\mathfrak{U}_{0, p}^{ \pm}(N), \mathfrak{U}_{0, p}(N)$ обозначим двухсторонние идеалы в $\mathfrak{U}_{p}(N)$, порожденные ПДО класса $O P \mathcal{T}_{1,0}^{ \pm}(N), O P \mathcal{T}_{1,0}(N) \cap O P S_{1,0}^{-1}(N)$. Положим

$$
\widehat{\mathfrak{U}}_{p}^{ \pm}(N)=\mathfrak{U}_{p}(N) \backslash \mathfrak{U}_{0, p}^{ \pm}(N), \quad \tilde{\mathfrak{U}}_{p}(N)=\mathfrak{U}_{p}(N) \backslash \mathfrak{U}_{0, p}(N) .
$$

Заметим, что $\mathfrak{U}_{0, p}(N)=\mathfrak{K}\left(L_{p}^{N}\right)$.

Пусть $A \in \mathfrak{U}_{p}^{ \pm}(N) \quad\left(A \in \mathfrak{U}_{p}(N)\right)$ и $O P \widetilde{S}(N) \ni A_{n} \rightarrow A$ в $\mathfrak{L}\left(L_{p}^{N}\right)$. Тогда в силу оценки $(1.21)\left(\left(1.21^{\prime}\right)\right)$ последовательность $a_{n}(x, \xi)$ фундаментальна по норме $\|\cdot\|_{\widetilde{\mathcal{M}}_{p}^{ \pm}}\left(\left.|||\cdot|\right|_{p}\right)$ и, следовательно, факторпоследовательность $\hat{a}_{n}(x, \xi)$ сходится в $\widetilde{\mathscr{M}}_{p}^{ \pm}\left(\mathscr{M}_{p} /\left(\mathscr{M}_{p}^{0} \cap \mathcal{N}_{p}^{0}\right)\right)$ к некоторому пределу $\hat{\sigma}^{ \pm}(A)(\hat{\sigma}(A))$, который будем называть локальныцм символом $A \in \mathfrak{U}_{p}^{ \pm}$в точке $\pm \infty$ (главным символом $A \in \mathfrak{U}_{p}(N)$ ).

Из теоремы 1.3 вытекают следуюшие оценки:

$$
\begin{gathered}
\left\|\hat{\sigma}^{ \pm}(A)\right\|_{\mathscr{M}_{p}^{ \pm}} \leqslant\|\hat{A}\|_{\widehat{\mathfrak{U}}_{p}^{ \pm}} \leqslant C\left\|\hat{\sigma}^{ \pm}(A)\right\|_{\mathscr{M}_{p}^{ \pm}}, \\
\left\|\left|\hat{\sigma}(A)\|\|_{p} \leqslant\|\hat{A}\|_{\tilde{U}_{p}} \leqslant C\right|\right\| \hat{\sigma}(A)\|\|_{p}
\end{gathered}
$$

где $\hat{A}$ - факторкласс, отвечаюший $A$.

Пусть $m_{p}^{ \pm}(N)\left(m_{p}(N)\right)$ - замыкание в $\mathscr{M}_{p}^{ \pm}(N) \quad\left(\mathscr{M}_{p}(N) /\left(\mathscr{M}_{p}^{0}(N) \cap \mathscr{N}_{p}^{0}(N)\right)\right)$ класса символов $O P \widetilde{S}(N)$. Тогда очевидно, что отображение $\Sigma_{ \pm}: \mathfrak{U}_{p}^{ \pm}(N) \rightarrow$ $m_{p}^{ \pm}(N)\left(\Sigma: \mathfrak{U}_{p} \rightarrow m_{p}\right)$, действуюшее по формуле $\Sigma_{ \pm}(A)=\hat{\sigma}^{ \pm}(A)(\Sigma(A)=\hat{\sigma}(A))$, есть изоморфизм банаховых алгебр.

Следствием теоремы 1.1 являются следуюшие теоремы. 
Tеорема 1.4. Пусть $A \in \mathfrak{U}_{p}(N), \quad p \in(1, \infty)$. Тогда следующие утверждения равносильны:

(a) $A: L_{p}^{N} \rightarrow L_{p}^{N}$ локально обратим в точке $+\infty$;

(b) локальный символ $\hat{\sigma}_{A}^{+}(x, \xi)$ оператора $A$ обратим в факторалгебре $\widetilde{\mathscr{M}}_{p}^{+}(N)$, что равносильно тому, ито

$$
\lim _{R \rightarrow+\infty} \inf _{(y, \xi) \in(R,+\infty) \times \mathbb{R}}\left|\operatorname{det} \sigma_{A}^{+}(x, \xi)\right|>0,
$$

где $\sigma_{A}^{+}(x, \xi)$ - некоторый представитель факторкласса $\hat{\sigma}_{A}^{+}(x, \xi)$.

Аналогичным образом формулируется утверждение о локальной обратимости в точке $-\infty$ для оператора из банаховой алгебры $\mathfrak{U}_{p}^{-}(N)$.

ДокАЗАТЕЛЬСТво. (а) $\Rightarrow(\mathrm{b})$. Если $A: L_{p}^{N} \rightarrow L_{p}^{N}$ локально обратим слева в точке $+\infty$, то верна априорная оценка $(1.12)$, из которой так же, как и при доказательстве теоремы 1.1, вытекает условие (b). Из (b) следует, что факторкласс $\hat{A} \in \widehat{\mathfrak{U}}_{p}^{+}(N)$, отвечаюший $A$, обратим в $\widehat{\mathfrak{U}}_{p}^{+}(N)$, т.е. сушествуют операторы $B^{\prime}, B^{\prime \prime} \in \mathfrak{U}_{p}^{+}(N)$ такие, что

$$
B^{\prime} A=I+T^{\prime}, \quad A B^{\prime \prime}=I+T^{\prime \prime}, \quad \text { где } T^{\prime}, T^{\prime \prime} \in \mathfrak{U}_{0, p}^{+}(N) .
$$

Пусть $\varphi_{R}(x)$ и $\psi_{R}(x)$ - срезаюшие функции окрестности точки $+\infty$ такие, что $\varphi_{R}(x) \psi_{R}(x)=\psi_{R}(x)$. Выберем $R>0$ так, чтобы $\left\|T^{\prime} \varphi_{R}\right\|<1 / 2,\left\|\varphi_{R} T^{\prime \prime}\right\|<1 / 2$; тогда $\mathfrak{B}^{\prime}=\left(I+T^{\prime} \varphi_{R}\right)^{-1} B^{\prime}, \mathfrak{B}^{\prime \prime}=B^{\prime \prime}\left(I+\varphi_{R} T^{\prime \prime}\right)^{-1} \in \mathfrak{U}_{p}^{+}$и являются левыми и правыми обратными операторами $A \in \mathfrak{U}_{p}^{+}(N)$.

Теорема 1.5. Пусть $A \in \mathfrak{U}_{p}(N), \quad p \in(1, \infty)$. Тогда следующие утверждения равносильны:

(a) $A: L_{p}^{N} \rightarrow L_{p}^{N}$ фредгольмов;

(b) главный символ $\hat{\sigma}_{A}(x, \xi)$ оператора $A$ обратим в факторалгебре $\mathscr{M}_{p}(N) /\left(\mathscr{M}_{p}^{0}(N) \cap \mathscr{N}_{p}^{0}(N)\right)$, что равносильно тому, что

$$
\lim _{R \rightarrow+\infty} \inf _{|x|+|\xi|>R}\left|\operatorname{det} \sigma_{A}(x, \xi)\right|>0,
$$

где $\sigma_{A}(x, \xi)$ - некоторый представитель факторкласса $\hat{\sigma}_{A}(x, \xi)$.

Индекс фредгольмового оператора $A \in \mathfrak{U}_{p}(N)$ вычисляется по формуле (1.19), в которой $a(x, \xi)$ следует заменить на $\sigma_{A}(x, \xi)$.

Предложение 1.6 так же очевидным образом переносится на операторы из алгебры $\mathfrak{U}_{p}(N)$.

\section{§ 2. Псевдодифференциальные операторы Меллина}

1. Исчисление. Через $L_{p, \delta}^{N}=L_{p, \delta}\left(\mathbb{R}_{+}, \mathbb{C}^{N}\right), p \in(1, \infty), \delta \in \mathbb{R}$, обозначается банахово пространство комплексных вектор-функций $u(r)=\left(u_{1}(r), \ldots, u_{N}(r)\right)$ с нормой

$$
\|u\|_{L_{p, \delta}^{N}}=\left(\int_{\mathbb{R}_{+}} r^{p \delta} \sum_{j=1}^{N}\left|u_{j}(r)\right|^{p} d r\right)^{1 / p} .
$$

Положим $\mathbb{R}_{\mu}=\{z \in \mathbb{C}: \operatorname{Im} z=\mu\}$. 
ОПРЕДЕЛЕНИЕ 2.1. Будем говорить, что матрица-функиия $a(r, \lambda)$ принадлежит классу $\mathscr{E}^{m}\left(\mathbb{R}_{+} \times \mathbb{R}_{\mu}, \mathbb{C}^{N \times N}\right)$, если $a(r, \lambda)=\left(a_{i, j}(r, \lambda)\right)_{i, j=1}^{N}$, где функции $a_{i, j}(r, \lambda)$ бесконечно дифференцируемы на $\mathbb{R}_{+} \times \mathbb{R}_{\mu}$ и удовлетворяют оценкам

$$
\sup _{r \in \mathbb{R}_{+}, \lambda \in \mathbb{R}_{\mu}}\left|\left(r \partial_{r}\right)^{\beta} \partial_{\lambda}^{\alpha} a_{i, j}(r, \lambda)\right|\langle\lambda\rangle^{-m+\alpha}<\infty
$$

для любых чисел $\beta, \alpha \in \mathbb{Z}_{+}$.

Будем говорить, что матрица функиия $a(r, \lambda) \in \mathscr{E}^{0}\left(\mathbb{R}_{+} \times \mathbb{R}_{\mu}, \mathbb{C}^{N \times N}\right)$ медленно меняется в 0, на $+\infty$, если соответственно

$$
\begin{aligned}
\lim _{r \rightarrow+0} \sup _{\lambda \in \mathbb{R}_{\mu}}\left|\left(r \partial_{r}\right)^{\beta} \partial_{\lambda}^{\alpha} a_{i, j}(r, \lambda)\right|\langle\lambda\rangle^{\alpha}=0, \\
r \rightarrow+\infty \sup _{\lambda \in \mathbb{R}_{\mu}}\left|\left(r \partial_{r}\right)^{\beta} \partial_{\lambda}^{\alpha} a_{i, j}(r, \lambda)\right|\langle\lambda\rangle^{\alpha}=0
\end{aligned}
$$

$\forall \beta \neq 0, \forall \alpha \in \mathbb{Z}_{+}$.

Класс медленно меняющихся как в 0 , так и на $+\infty$ матрищ-функций обозначим через $\widetilde{\mathscr{E}}\left(\mathbb{R}_{+} \times \mathbb{R}_{\mu}, \mathbb{C}^{N \times N}\right)$. Если соответственно

$$
\lim _{r \rightarrow+0} \sup _{\lambda \in \mathbb{R}_{\mu}}\left|a_{i, j}(r, \lambda)\right|=0, \quad \lim _{r \rightarrow+\infty} \sup _{\lambda \in \mathbb{R}_{\mu}}\left|a_{i, j}(r, \lambda)\right|=0,
$$

то будем говорить, что $a(r, \lambda)$ принадлежит классу $\mathscr{K}_{0}\left(\mathbb{R}_{+} \times \mathbb{R}_{\mu}, \mathbb{C}^{N \times N}\right)$ или $\mathscr{K}_{+\infty}\left(\mathbb{R}_{+} \times \mathbb{R}_{\mu}, \mathbb{C}^{N \times N}\right)$.

Положим $\mathscr{K}\left(\mathbb{R}_{+} \times \mathbb{R}_{\mu}, \mathbb{C}^{N \times N}\right)=\mathscr{K}_{0}\left(\mathbb{R}_{+} \times \mathbb{R}_{\mu}, \mathbb{C}^{N \times N}\right) \cap \mathscr{K}_{+\infty}\left(\mathbb{R}_{+} \times \mathbb{R}_{\mu}, \mathbb{C}^{N \times N}\right)$. Матрице-функции $a(r, \lambda) \in \mathscr{E}\left(\mathbb{R}_{+} \times \mathbb{R}_{\mu}, \mathbb{C}^{N \times N}\right)$, называемой символом, сопоставим ПДО Меллина

$$
(A u)(x)=a\left(r, \mathscr{D}_{r}\right)=(2 \pi)^{-1} \iint_{\mathbb{R}_{+} \times \mathbb{R}_{\mu}} a(r, \lambda)\left(r \rho^{-1}\right)^{i \lambda} u(\rho) \rho^{-1} d \rho d \lambda
$$

где $u(\rho) \in C_{0}^{\infty}\left(\mathbb{R}_{+}, \mathbb{C}^{N}\right)$. Интеграл в формуле $(2.2)$ будем понимать как повторный либо как осциллирующий при естественной модификации этого понятия. Через $O P \mathscr{E}_{\mu}(N), O P \widetilde{\mathscr{E}}_{\mu}(N), O P \mathscr{K}_{0, \mu}(N), O P \mathscr{K}_{+\infty, \mu}(N), O P \mathscr{K}_{\mu}(N)$ будем обозначать соответствуюшие классы ПДО Меллина.

ОПРЕДЕЛЕНИЕ 2.2. Будем говорить, что оператор-функция $a(r, \rho, \lambda)$ принадлежит классу $\mathscr{E}^{0}\left(\mathbb{R}_{+}^{2} \times \mathbb{R}_{\mu}, \mathbb{C}^{N \times N}\right)$, если $a(r, \rho, \lambda)$ - бесконечно дифференщируемая матрица-функция на $\mathbb{R}_{+}^{2} \times \mathbb{R}_{\mu}$ и ее компоненты $a_{i, j}(r, \rho, \lambda)$ удовлетворяют оценкам

$$
\sup _{r \in \mathbb{R}, \rho \in \mathbb{R}, \lambda \in \mathbb{R}_{\mu}}\left|\left(r \partial_{r}\right)^{\beta}\left(\rho \partial_{\rho}\right)^{\gamma} \partial_{\lambda}^{\alpha} a_{i, j}(r, \rho, \lambda)\right|\langle\lambda\rangle^{\alpha}<C_{\alpha \beta \gamma}
$$

для любых чисел $\alpha, \beta, \gamma \in \mathbb{Z}_{+}, i, j=1, \ldots, N$.

Будем говорить, что $a(r, \rho, \lambda)$ медленно меняется, если одновременно

$$
\begin{aligned}
& \lim _{r \rightarrow+0} \sup _{\rho \in K, \lambda \in \mathbb{R}_{\mu}}\left|\left(r \partial_{r}\right)^{\beta}\left(\rho \partial_{\rho}\right)^{\gamma} \partial^{\alpha} a_{i, j}(r, \rho r, \lambda)\right|\langle\lambda\rangle^{\alpha}=0, \\
& \lim _{r \rightarrow+\infty} \sup _{\rho \in K, \lambda \in \mathbb{R}_{\mu}}\left|\left(r \partial_{r}\right)^{\beta}\left(\rho \partial_{\rho}\right)^{\gamma} \partial^{\alpha} a_{i, j}(r, \rho r, \lambda)\right|\langle\lambda\rangle^{\alpha}=0
\end{aligned}
$$


при $\beta+\gamma \neq 0 \forall \alpha, \forall i, j$, где $K$ - произвольный компакт в $\mathbb{R}_{+}$.

Оператор

$$
(A u)(x)=(2 \pi)^{-1} \iint_{\mathbb{R}_{+} \times \mathbb{R}_{\mu}} a(r, \rho, \lambda)\left(r \rho^{-1}\right)^{i \lambda} u(\rho) \rho^{-1} d \rho d \lambda,
$$

где $u(\rho) \in C_{0}^{\infty}\left(\mathbb{R}_{+}, \mathbb{C}^{N}\right)$, называется ПДО Меллина с двойньлм символом.

Интеграл в формулах (2.2), (2.4) понимается как осциллирующий при естественной модификации этого определения, состоящей в замене оператора $\langle D\rangle$ на $\left\langle\rho \partial_{\rho}\right\rangle$, а $\langle y\rangle$ на $\langle\ln \rho\rangle$.

С помощью стандартной замены переменных $r=e^{-x}, \rho=e^{-y}$ ПДО Меллина трансформируются в матричные ПДО на прямой, рассмотренные в предыдущем параграфе, причем точка $+\infty$ переходит в точку 0 , а точка $-\infty$ в $-\infty$, поэтому результаты $\S 1$ очевидным образом переносятся на ПДО Меллина.

Приведем необходимые в дальнейшем результаты.

ПРЕДЛОЖЕНИЕ 2.1. (а) Если $A \in O_{\delta+1 / p}^{\mathscr{0}}(N)$, то $A$ ограничен $в$ $L_{p, \delta}\left(\mathbb{R}_{+}, \mathbb{C}^{N}\right) u\|A u\|_{L_{p, \delta}^{N}} \leqslant M\|u\|_{L_{\rho, \delta}^{N}}$ c константой $M$, определяемой, как и в предложсении 1.3, с заменой операторов дифференцирования по $x, y$ соответственно операторами дифференцирования $t \partial_{t}, \tau \partial_{\tau}$.

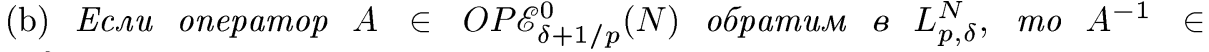
$O P \mathscr{E}_{\delta+1 / p}^{0}(N)$.

(c) $E c \bumpeq u \quad A, B \in O P \widetilde{\mathscr{E}}_{\delta+1 / p}(N)$, mо $A B \in O P \widetilde{\mathscr{E}}_{\delta+1 / p}(N)$ и символ $\sigma_{A B}(x, \xi)$ оператора $A B$ вычисляется по формуле

$$
\sigma_{A B}(r, \lambda)=b(r, \lambda) a(r, \lambda)+t(r, \lambda),
$$

əде $t(r, \lambda) \in \mathscr{E}^{-1}\left(\mathbb{R}_{+} \times \mathbb{R}_{\delta+1 / p}, \mathbb{C}^{N \times N}\right) \cap \mathscr{K}\left(\mathbb{R}_{+} \times \mathbb{R}_{\delta+1 / p}, \mathbb{C}^{N \times N}\right)$.

(d) Ecли $A \in O P \widetilde{\mathscr{E}}_{\delta+1 / p}(N)$ и действует в $L_{p, \delta}^{N}$, mo $A^{*} \in O P \widetilde{\mathscr{E}}_{\delta+1 / p}(N) u$ его символ вычисляется по формуле

$$
\sigma_{A^{*}}(r, \lambda)=a^{*}(r, \lambda)+t(r, \lambda),
$$

где $t(r, \lambda) \in \mathscr{E}^{-1}\left(\mathbb{R}_{+} \times \mathbb{R}_{\delta+1 / p}, \mathbb{C}^{N \times N}\right) \cap \mathscr{K} /\left(\mathbb{R}_{+} \times \mathbb{R}_{\delta+1 / p}, \mathbb{C}^{N \times N}\right)$.

(е) Если $а(r, \rho, \lambda) \in \widetilde{\mathscr{E}}\left(\mathbb{R}_{+}^{2} \times \mathbb{R}_{\delta+1 / p}, \mathbb{C}^{N \times N}\right)$ и $A$ - соответствующий ПДО с двойным символом, то

$$
\sigma_{A}(r, \lambda)=a(r, r, \lambda)+t(r, \lambda),
$$

əде $t(r, \lambda) \in \mathscr{E}^{-1}\left(\mathbb{R}_{+} \times \mathbb{R}_{\delta+1 / p}, \mathbb{C}^{N \times N}\right) \cap \mathscr{K}\left(\mathbb{R}_{+} \times \mathbb{R}_{\delta+1 / p}, \mathbb{C}^{N \times N}\right)$.

2. Локальная обратимость. Пусть $A=a\left(r, \mathscr{D}_{r}\right) \in O P \widetilde{\mathscr{E}}_{\delta+1 / p}(N)$. Введем числа

$$
\begin{aligned}
d_{p, \delta}^{0}(a) & =\lim _{\varepsilon \rightarrow+0} \inf _{(0, \varepsilon) \times \mathbb{R}_{\delta+1 / p}}|\operatorname{det} a(r, \lambda)|, \\
d_{p, \delta}^{+\infty}(a) & =\lim _{\varepsilon \rightarrow+\infty} \inf _{(\tau,+\infty) \times \mathbb{R}_{\delta+1 / p}}|\operatorname{det} a(r, \lambda)| .
\end{aligned}
$$

Если $A-\Pi$ ПО Меллина с двойным символом, то числа $d_{p, \delta}^{0}(a), d_{p, \delta}^{+\infty}(a)$ определяются по символу $a(r, r, \lambda)$. 
Teорема 2.1. Пусть $A=a\left(r, \mathscr{D}_{r}\right) \in O \widetilde{\mathscr{E}}_{\delta+1 / p}(N)$. Тогда следующие условия равносильны:

(a) оператор $A: L_{p, \delta}\left(\mathbb{R}_{+}, \mathbb{C}^{N}\right) \rightarrow L_{p, \delta}\left(\mathbb{R}_{+}, \mathbb{C}^{N}\right)$ локально обратим в точке нуль;

(b) $d_{p, \delta}^{0}(a)>0$;

(c) существуют левый и правый локально обратные операторы в точке 0 , принадлежащие $О \widetilde{\mathscr{\mathscr { E }}}_{\delta+1 / p}(N)$.

Аналогичным образом формулируется условие локальной обратимости в точке $+\infty$.

\section{3. Фредгольмовость и индекс.}

Teоpema 2.2. Пусть $A=a\left(r, \mathscr{D}_{r}\right) \in O \mathscr{P}_{\delta+1 / p}(N)$. Тогда $a\left(r, \mathscr{D}_{r}\right): L_{p, \delta}\left(\mathbb{R}_{+}\right.$, $\left.\mathbb{C}^{N}\right) \rightarrow L_{p, \delta}\left(\mathbb{R}_{+}, \mathbb{C}^{N}\right)$ фредгольмов тогда и только тогда, когда:

(a) $\quad d_{p, \delta}^{0}(a)>0$

(b) $d_{p, \delta}^{+\infty}(a)>0$;

(c) $\quad d_{p, \delta}^{\prime}(a)=\lim _{t \rightarrow \infty} \inf _{\mathbb{R}_{+} \times\{\mu:|\mu|>t\}}|\operatorname{det} a(r, \mu+i(\delta+1 / p))|>0$.

Если условия (a), (b) и (c) выполненьь, то

$$
\text { ind } a\left(r, \mathscr{D}_{r}\right)=-(2 \pi)^{-1}[\arg \operatorname{det} a(r, \mu+i(\delta+1 / p))]_{\Gamma\left(R^{\prime}, R^{\prime \prime}\right)},
$$

где $\Gamma\left(R^{\prime}, R^{\prime \prime}\right)$ - положительно ориентированная гранича прямоугольника $\Pi\left(R^{\prime}, R^{\prime \prime}\right)=\left\{(r, \mu) \in \mathbb{R}^{2}: 1 / R^{\prime}<r<R^{\prime},|\mu|<R^{\prime \prime}\right\}$, а числа $R^{\prime}, R^{\prime \prime}$ настолько велики, что в области $\mathbb{R}^{2} \backslash \Pi\left(R^{\prime}, R^{\prime \prime}\right)$ существует $a^{-1}(r, \mu+i(\delta+1 / p))$.

Пусть $\chi_{\alpha}^{+}(r)$ - характеристическая функция интервала $\mathbb{L}_{\alpha}=(0, \alpha), \chi_{\alpha}^{-}(r)=$ $1-\chi_{\alpha}^{+}(r)$. Справедлив аналог предложения 1.6.

ПРЕДЛОЖЕНИЕ 2.2. Пусть $A=a\left(r, \mathscr{D}_{r}\right) \in O \widetilde{\mathscr{\mathscr { E }}}_{\delta+1 / p}(N)$ и его символ $a(r, \lambda)$ тождественно равен 1 при $r \geqslant a-\varepsilon(\varepsilon>0)$. Оператор $\chi_{\alpha}^{+} A \chi_{\alpha}^{+}$фредгольмов в $L_{p, \delta}\left(\mathbb{L}_{\alpha}, \mathbb{C}^{N}\right)$ тогда и только тогда, когда оператор А фредгольмов в $L_{p, \delta}\left(\mathbb{R}_{+}, \mathbb{C}^{N}\right)$, причем индексы этих операторов совпадают.

4. Банаховы алгебры $\mathfrak{N}_{p, \delta}(N), \mathscr{R}_{p, \delta}(N), r_{p, \delta}(N)$.

ОПРЕДЕЛЕНИЕ 2.3. Через $\mathfrak{N}_{p, \delta}(N)$ будем обозначать банахову алгебру а $(\lambda)$ меллиновских мультипликаторов в $L_{p, \delta}\left(\mathbb{R}_{+}, \mathbb{C}^{N}\right)$, т.е. символов ПДО Меллина $a(\mathscr{D})$, инвариантных относительно мультипликативного сдвига и ограниченных в $L_{p, \delta}\left(\mathbb{R}_{+}, \mathbb{C}^{N}\right)$.

Норма в $\mathfrak{N}_{p, \delta}(N)$ вводится по формуле

$$
\|a(\cdot)\|_{\mathfrak{N}_{p, \delta}(N)}=\|a(\mathscr{D})\|_{\mathfrak{L}_{\left(L_{p, \delta}\left(\mathbb{R}_{+}, \mathbb{C}^{N}\right)\right)}, \quad 1<p<\infty .}
$$

Через $\mathscr{R}_{p, \delta}(N)$ обозначим банахову алгебру ограниченных, непрерывных на $\mathbb{R}_{+}$матрич-функций $a(r, \lambda)$ со значениями в $\mathfrak{N}_{p, \delta}(N)$, нормой

$$
\|a\|_{\mathscr{R}_{p, \delta}}=\sup _{r \in \mathbb{R}_{+}}\|a(r, \cdot)\|_{\mathfrak{N}_{p, \delta}(N)}
$$

и естественной операцией умножения матриц-функций.

Через $r_{p, \delta}(N)$ обозначим замыкание в $\mathscr{R}_{p, \delta}(N)$ класса символов $\widetilde{\mathscr{E}}\left(\mathbb{R}_{+}^{2} \times\right.$ $\left.\mathbb{R}_{\delta+1 / p}, \mathbb{C}^{N \times N}\right)$. 
ЗАмЕчАниЕ. Нетрудно показать, что алгебра $r_{p, \delta}(N)$ порождена инвариантными относительно подобия ПДО Меллина классами $O P \widetilde{\mathscr{E}}_{\delta+1 / p}\left(\mathbb{C}^{N}\right)$ и операторами умножения на матрицы-функции класса $S V\left(\mathbb{R}_{+}, N\right)$, где $S V\left(\mathbb{R}_{+}, N\right)$ состоит из матриц-функций, элементы которых есть замыкания в $C_{b}\left(\mathbb{R}_{+}\right)$множества таких функций $a(r) \in C_{b}\left(\mathbb{R}_{+}\right)$, что

$$
\lim _{r \rightarrow+0} r a^{\prime}(r)=\lim _{r \rightarrow+\infty} r a^{\prime}(r)=0
$$

Через $\mathfrak{I}_{p, \delta}^{0}(N), \mathfrak{I}_{p, \delta}^{+\infty}(N)$ обозначим идеалы в $\mathscr{R}_{p, \delta}(N)$, состояшие из таких функций $a \in \mathscr{R}_{p, \delta}(N)$, что соответственно

$$
\lim _{r \rightarrow+0}\|a(r, \cdot)\|_{\mathfrak{N}_{p, \delta}(N)}=0, \quad \lim _{r \rightarrow+\infty}\|a(r, \cdot)\|_{\mathfrak{N}_{p, \delta}(N)}=0 .
$$

Положим $\mathfrak{I}_{p, \delta}(N)=\mathfrak{I}_{p, \delta}^{0}(N) \cap \mathfrak{I}_{p, \delta}^{+\infty}(N)$ и введем еше один идеал $\mathfrak{T}_{p, \delta}(N)$ в $\mathscr{R}_{p, \delta}(N)$, состоящий из таких функций, что

$$
\lim _{\mathbb{R}_{\delta+1 / p} \ni \lambda \rightarrow \infty} \sup _{r \in \mathbb{R}_{+}}\|a(r, \lambda)\|_{p}=0
$$

Пусть

$$
\begin{gathered}
\mathscr{R}_{p, \delta}^{+0}(N)=\mathscr{R}_{p, \delta}(N) / \mathfrak{I}_{p, \delta}^{0}(N), \quad \mathscr{R}_{p, \delta}^{+\infty}(N)=\mathscr{R}_{p, \delta}(N) / \mathfrak{I}_{p, \delta}^{+\infty}(N), \\
\widetilde{\mathscr{R}}_{p, \delta}(N)=\mathscr{R}_{p, \delta}(N) / \mathfrak{I}_{p, \delta}(N) \cap \mathfrak{T}_{p, \delta}(N)
\end{gathered}
$$

- соответствуюшие факторалгебры с естественной нормой.

Через $r_{p, \delta}^{+}(N), \tilde{r}_{p, \delta}(N)$ обозначим соответственно образ $r_{p, \delta}(N)$ при естественном отображении $\mathscr{R}_{p, \delta}(N)$ в $\mathscr{R}_{p, \delta}^{+}(N)$ и $\mathscr{R}_{p, \delta}(N)$ в $\widetilde{\mathscr{R}}_{p, \delta}(N)$.

Отметим некоторые свойства введенных выше банаховых алгебр:

$$
\begin{gathered}
\mathscr{R}_{p, \delta}(N)=\mathscr{R}_{q, \delta}(N), \quad p^{-1}+q^{-1}=1, \quad 1<p<\infty \\
\mathscr{R}_{p, \delta}(N) \subset \mathscr{R}_{2, \delta}(N)
\end{gathered}
$$

и это вложение топологическое; причем алгебра $\mathscr{R}_{2, \delta}(N)$ изометрически изоморфна алгебре $C_{b}\left(\mathbb{R}_{+}, L_{\infty}\left(\mathbb{R}_{\delta+1 / p}, \mathfrak{L}\left(\mathbb{C}^{N}\right)\right)\right.$, где $C_{b}\left(\mathbb{R}_{+}, L_{\infty}\left(\mathbb{R}_{\mu}, \mathfrak{L}\left(\mathbb{C}^{N}\right)\right)\right)$ - замыкание алгебраического тензорного произведения $\left.C_{b}\left(\mathbb{R}_{+}\right) \otimes L_{\infty}\left(\mathbb{R}_{\mu}, \mathfrak{L}\left(\mathbb{C}^{N}\right)\right)\right)$ по норме

$$
\|a\|=\sup _{r \in \mathbb{R}}\|a(r, \cdot)\|_{L_{\infty}\left(\mathbb{R}_{\mu}, \mathfrak{L}\left(\mathbb{C}^{N}\right)\right)}
$$

(в $\mathfrak{L}\left(\mathbb{C}^{N}\right)$ вводится евклидова норма).

Аналогичными свойствами обладают факторалгебры $\mathscr{R}_{p, \delta}^{+0}(N), \mathscr{R}_{p, \delta}^{+\infty}(N)$, $\widetilde{\mathscr{R}}_{p, \delta}(N), r_{p, \delta}^{+0}(N), \tilde{r}_{p, \delta}(N)$. 
ПРЕДЛОЖЕНИЕ 2.3. Факторкласс $\hat{a} \in \mathscr{R}_{p, \delta}^{+}(N) \quad\left(\hat{a} \in \widetilde{\mathscr{R}}_{p, \delta}(N)\right), \quad 1<p<\infty$, обратим в $\mathscr{R}_{p, \delta}^{+}(N) \quad\left(\widetilde{\mathscr{R}}_{p, \delta}(N)\right)$ тогда и только тогда, когда

$$
\begin{aligned}
& d_{p, \delta}(a)=\lim _{R \rightarrow+0} \inf _{(r, \lambda) \in(0, R) \times \mathbb{R}_{\delta+1 / p}}|\operatorname{det} a(r, \lambda)|>0, \\
& d_{p, \delta}^{\prime}(a)=\lim _{R \rightarrow+\infty}(r, \lambda) \in(1 / R, R) \times \mathbb{R}_{\delta+1 / p}|\operatorname{det} a(r, \lambda)|>0, \\
& d_{p, \delta}^{\prime \prime}(a)=\lim _{R \rightarrow+\infty}(r, \lambda) \in \mathbb{R}_{+} \times(-R, R)+i(\delta+1 / p)
\end{aligned}
$$

әде а - произвольный представитель факторкласса $\hat{a}$.

Справедлива следуюшая теорема, вытекающая из теорем $1.3,1.3^{\prime}$.

Teopema 2.3. Пусть $A \in O P \mathscr{E}_{\delta+1 / p}(N), \quad 1<p<\infty$. Тогда

$$
\begin{aligned}
\|\hat{a}\|_{\mathscr{R}_{p, \delta}^{+}(N)} & \leqslant \inf _{T \in O P \mathscr{K}_{0, \delta+1 / 2}(N)}\|A-T\|_{\mathcal{L}\left(L_{p, \delta}^{N}\right)} \leqslant C\|\hat{a}\|_{\mathscr{R}_{p, \delta}^{+}(N)}, \\
\|\hat{a}\|_{\widetilde{\mathscr{R}}_{p, \delta}(N)} & \leqslant \inf _{T \in \mathfrak{K}\left(L_{p, \delta}^{N}\right)}\|A-T\|_{\mathcal{L}\left(L_{p, \delta}^{N}\right)} \leqslant C\|\hat{a}\|_{\widetilde{\mathscr{R}}_{p, \delta}(N)}
\end{aligned}
$$

с константой $C>0$, не зависящей от $A, p, \delta$. При $p=2$ константа $C$ равна 1 .

5. Банахова алгебра $\mathscr{V}_{p, \delta}(N)$. Через $\mathscr{V}_{p, \delta}(N)$ обозначим банахову алгебру операторов, действуюших в пространстве $L_{p, \delta}\left(\mathbb{R}_{+}, \mathbb{C}^{N}\right), p \in(1, \infty)$, полученную замыканием в $\mathfrak{L}\left(L_{p, \delta}\left(\mathbb{R}_{+}, \mathbb{C}^{N}\right)\right)$ множества псевдодифференциальных операторов $a(r, \mathscr{D}) \in O P \mathscr{E}_{\delta+1 / p}$. Через $\mathscr{V}_{p, \delta}^{0}(N)$ обозначим двухсторонний идеал в $\mathscr{V}_{p, \delta}(N)$, порожденный псевдодифференциальными операторами класса

$O P \mathscr{K}_{0, \delta+1 / p}(N), \quad \widehat{\mathscr{V}}_{p, \delta}^{0}(N)=\mathscr{V}_{p, \delta}(N) / \mathscr{V}_{p, \delta}^{0}(N), \quad \widehat{\mathscr{V}}_{p, \delta}(N)=\mathscr{V}_{p, \delta}(N) / \mathfrak{K}\left(L_{p, \delta}^{N}\right)$.

Как и в предыдущем параграфе, предельньй переход и теорема 2.2 позволяют сопоставить каждому оператору $A \in \mathscr{V}_{p, \delta}(N)$ его локальный символ $\hat{\sigma}^{0}(A)$ в точке 0 как элемент факторалгебры $r_{p, \delta}^{+0}(N)$ и глобальный символ $\hat{\sigma}(A)$ как элемент факторалгебры $\tilde{r}_{p, \delta}(N)$, причем справедливы оценки

$$
\begin{aligned}
\left\|\hat{\sigma}^{0}(A)\right\|_{\mathscr{R}_{p, \delta}^{+}(N)} & \leqslant\|\hat{A}\|_{\widehat{\mathcal{V}}_{p, \delta}} \leqslant C\left\|\hat{\sigma}^{0}(A)\right\|_{\mathscr{R}_{p, \delta}^{+}(N)}, \\
\|\hat{\sigma}(A)\|_{\widetilde{\mathscr{R}}_{p, \delta}(N)} & \leqslant\|\hat{A}\|_{\mathfrak{L}\left(L_{p, \delta}^{N}\right) / \mathfrak{K}\left(L_{p, \delta}^{N}\right)} \leqslant C\left\|\hat{\sigma}^{0}(A)\right\|_{\widetilde{\mathscr{R}}_{p, \delta}(N)},
\end{aligned}
$$

где $\hat{A}$ - факторкласс, отвечаюший оператору $A$.

Теорема 2.4. Отображения

$$
\Sigma_{+}: \widehat{\mathscr{V}}_{p, \delta}(N) \rightarrow r_{p, \delta}^{+}(N), \quad \Sigma: \widehat{\mathscr{V}}_{p, \delta}(N) \rightarrow \tilde{r}_{p, \delta}(N),
$$

действующие по формулам

$$
\Sigma_{+}(\hat{A})=\hat{\sigma}^{0}(A), \quad \Sigma(\hat{A})=\hat{\sigma}(A),
$$

являются изоморфизмами банаховых алгебр, изометрическими в случае $p=2$. 
Tеорема 2.5. Пусть $A \in \mathscr{V}_{p, \delta}(N), \quad p \in(1, \infty)$. Тогда следующие утверждения равносильны:

(a) $A: L_{p, \delta}\left(\mathbb{R}_{+}, \mathbb{C}^{N}\right) \rightarrow L_{p, \delta}\left(\mathbb{R}_{+}, \mathbb{C}^{N}\right)$ локально обратим в точке нуль;

(b) локальный символ $\hat{\sigma}_{A}^{0}(x, \xi)$ оператора $А$ обратим в факторалгебре $\mathscr{R}_{p, \delta}^{+}(N)$, что равносильно тому, что

$$
\lim _{R \rightarrow+0} \inf _{(r, \lambda) \in(0, R) \times \mathbb{R}_{\delta+1 / p}}\left|\operatorname{det} \sigma^{0}(A)(r, \lambda)\right|>0,
$$

где $\sigma^{0}(A)(r, \lambda)$ - некоторый представитель факторкласса $\hat{\sigma}^{0}(A)(r, \lambda)$.

Tеорема 2.5'. Пусть $A \in \mathscr{V}_{p, \delta}(N), \quad p \in(1, \infty)$. Тогда следующие утверждения равносильны:

(a) $A: L_{p, \delta}\left(\mathbb{R}_{+}, \mathbb{C}^{N}\right) \rightarrow L_{p, \delta}\left(\mathbb{R}_{+}, \mathbb{C}^{N}\right)$ фредгольмов;

(b) главный символ $\sigma(A)(x, \xi)$ оператора $A$ обратим в факторалгебре $\widetilde{\mathscr{R}}_{p, \delta}(N)$, что равносильно тому, что одновременно

$$
\begin{array}{r}
\lim _{R \rightarrow+\infty} \inf _{(r, \lambda) \in(1 / R, R) \times \mathbb{R}_{\delta+1 / p}}|\operatorname{det} \sigma(A)(r, \lambda)|>0, \\
\lim _{t \rightarrow \infty \mathbb{R}_{+} \times\{\mu:|\mu|>t\}}|\operatorname{det} \sigma(A)(r, \mu+i(\delta+1 / p))|>0,
\end{array}
$$

əде $\sigma(A)(r, \lambda)$ - некоторый представитель факторкласса $\hat{\sigma}(A)(r, \lambda)$.

Для вычисления индекса фредгольмова оператора $A \in \mathscr{V}_{p, \delta}(N)$ справедлива формула $(2.8)$, в которой $a(r, \lambda)$ заменено на $\sigma(A)(r, \lambda)$. Предложение 2.2 очевидным образом переносится на операторы класса $\mathscr{V}_{p, \delta}(N)$.

\section{§3. Банаховы алгебры сингулярных интегральных операторов на контурах с особенностями}

\section{1. Контур.}

ОПРЕДЕЛЕНИЕ 3.1. Будем говорить, что простая (гомеоморфная сегменту) спрямляемая незамкнутая дуга $\gamma \subset \mathbb{C}$ с конщами $x_{1}, x_{2}$ принадлежит классу $\mathfrak{R}_{\delta}$, если:

1) $\gamma \backslash\left\{x_{1}, x_{1}\right\}$ - локально-ляпуновская дуга;

2) пусть

$$
x-x_{j}=\varphi_{j}(s)=s \exp i \omega_{j}(s), \quad s \in\left[0, s_{j}\right],
$$

- параметризация $\gamma$ в окрестности конща $x_{j}$.

Будем предполагать, что $\omega_{j}(s)=\delta_{j} \ln s+\theta_{j}(s)$, где $\delta_{j} \in \mathbb{R}, \theta_{j}(s) \in C^{\infty}\left(\left(0, s_{j}\right]\right)$, и $\forall k \in \mathbb{N}$

$$
\lim _{s \rightarrow+0}(s \partial / \partial s)^{k} \theta_{j}(s)=0 .
$$

Типичными примерами функции $\theta(s)$, удовлетворяющей условию (3.1), являются

$$
|\ln s|^{\alpha}, \quad a+b \sin |\ln s|^{\alpha}, \quad a \in \mathbb{R}, \quad \alpha \in(0,1), \quad b \in \mathbb{R} .
$$

Заметим, что кривые $\gamma \in \mathfrak{R}_{\delta}$ имеют касательные в каждой точке открытой дуги, но при приближении к конщам они могут вращаться и осциллировать так, что в концах $x_{1}, x_{2}$ не существует односторонних касательных. 
ПРЕДЛОЖЕНИЕ 3.1. Пусть $\gamma \in \mathfrak{R}_{\delta} u$

$$
x-x_{0}=\varphi(s)=s \exp i(\delta \ln s+\theta(s))=s^{1+i \delta} \exp (i \theta(s))
$$

- параметризация $\gamma$ в некоторой окрестности конца $x_{0}$ кривой

$$
\gamma \in \mathfrak{R}_{\delta}, \quad \psi(s)=\exp (i \theta(s)) .
$$

Тогда:

(a) $\quad \lim _{s \rightarrow+0}(s \partial / \partial s)^{k} \psi(s)=0 \quad \forall k \in \mathbb{N}$;

(b) $\quad\left(1+\delta^{2}\right)^{-1 / 2}\left|t^{1+i \delta}-\tau^{1+i \delta}\right| \leqslant|\varphi(t)-\varphi(\tau)| \leqslant m\left|t^{1+i \delta}-\tau^{1+i \delta}\right|$

$$
\forall t, \tau \in\left(0, s_{0}\right], \quad m>0
$$

(c) $\quad|t-\tau| \leqslant|\varphi(t)-\varphi(\tau)| \leqslant m|t-\tau| \quad \forall t, \tau \in\left(0, s_{0}\right]$.

ДокАЗАТЕльство. (а) очевидно. Докажем оценку снизу в (3.3). Положим

$$
\varphi(t)=t \exp i \theta_{1}, \quad \varphi(\tau)=\tau \exp i \theta_{2}, \quad t>0, \quad \tau>0 .
$$

Тогда

$$
|\varphi(t)-\varphi(\tau)|=\left((t-\tau)^{2}+2 t \tau\left(1-\cos \left(\theta_{1}-\theta_{2}\right)\right)\right)^{1 / 2} \geqslant|t-\tau| .
$$

Оценка сверху в неравенстве (3.3), где $m=\sup _{t \in\left(0, s_{0}\right)}\left|\varphi^{\prime}(t)\right|$, очевидна. Докажем оценку сверху в (3.2). Используя (3.4), получаем

$$
|\varphi(t)-\varphi(\tau)| /\left|t^{1+i \delta}-\tau^{1+i \delta}\right| \leqslant|\varphi(t)-\varphi(\tau)| /|t-\tau| \leqslant m
$$

Докажем теперь оценку снизу в (3.2). Так как

$$
\left|t^{1+i \delta}-\tau^{1+i \delta}\right| \leqslant\left(1+\delta^{2}\right)^{1 / 2}|t-\tau|
$$

то

$$
|\varphi(t)-\varphi(\tau)| /\left|t^{1+i \delta}-\tau^{1+i \delta}\right| \geqslant\left(1+\delta^{2}\right)^{-1 / 2}|\varphi(t)-\varphi(\tau)| /|t-\tau| \geqslant\left(1+\delta^{2}\right)^{-1 / 2} .
$$

Пусть $Г$ - ориентированный контур в плоскости $\mathbb{C}$, состоящий из конечного числа дуг класса $\mathfrak{R}_{\delta}$. Предполагается, что любые две дуги могут пересекаться только в концевых точках, и пусть $F \subset \Gamma$ - множество концевых точек этих дуг. Точки множества $F$ будем называть узлами. Пусть $z \in F, N(z)$ - число дуг, составляющих узел $z$, и

$$
x-z=\varphi_{j}(s)=s \exp i \omega_{j}(s), \quad s \in\left[0, s_{j}\right], \quad j=1,2, \ldots, N(z),
$$

где $\omega_{j}(s)=\delta \ln s+\varkappa(s)+\theta_{j}(s), \delta \in \mathbb{R}$, а $\varkappa(s), \theta_{j}(s)(j=1, \ldots, N(z))$ удовлетворяют условию (3.1).

Будем предполагать, что сушествует $s_{0}>0$ такое, что $\theta_{j}(s) \in\left[\alpha_{j}, \beta_{j}\right], s \in$ $\left(0, s_{0}\right]$, где

$$
0 \leqslant \alpha_{1}<\beta_{1}<\alpha_{2}<\beta_{2}<\cdots<\alpha_{N}<\beta_{N}<2 \pi \text {. }
$$


ОПРЕДЕЛЕНИЕ 3.2. Через $P S V(\Gamma, F)$ обозначим $C^{*}$-алгебру, полученную замыканием по sup-норме на $\Gamma$ множества ограниченных, непрерывно дифференцируемых на $\Gamma \backslash F$ функций таких, что для любой точки $x_{j} \in F$

$$
\lim _{\Gamma \ni x \rightarrow x_{j}}\left|x-x_{j}\right| a^{\prime}(x)=0 .
$$

Функции класса $P S V(\Gamma, F)$ могут иметь ограниченные разрывы второго рода в окрестности узлов, и условие (3.7) есть условие медленной осциллящии в точках разрыва.

Пусть

$$
\omega(x)=\prod_{m=1}^{L}\left|x-x_{m}\right|^{\beta_{m}}, \quad x_{m} \in F, \quad \beta_{m} \in(-1 / p, 1-1 / p),
$$

- вес Хведелидзе на контуре $\Gamma$. Введем банахово пространство $L_{p}(\Gamma, \omega), \quad 1<$ $p<\infty$, с нормой

$$
\|u\|_{L_{p}(\Gamma, \omega)}=\left(\int_{\Gamma}(\omega(x)|u(x)|)^{p}|d x|\right)^{1 / p}
$$

Пусть

$$
S_{\Gamma}=(\pi i)^{-1} \int_{\Gamma} u(y)(y-x)^{-1} d y, \quad x \in \Gamma,
$$

- сингулярный интеграл на контуре $\Gamma$, ограниченный в $L_{p}(\Gamma, \omega)$, так как дуги $\gamma_{j}$, составляющие контур, удовлетворяют условию Карлесона [4]. Пусть $z \in F$. Реализуем $L_{p}(\Gamma, \omega)$ в окрестности узла $z$ как прямую сумму пространств $L_{p}\left(\gamma_{j}, \omega\right)$, $j=1, \ldots, N$, где $N=N(z), \gamma_{j}=\gamma_{j}(z)$, а оператор $S_{\Gamma}-$ как матричный операTop $\left(S_{j k}\right)_{j, k=1}^{N}$,

$$
\left(S_{j k} u\right)(x)=(\pi i)^{-1} \int_{\gamma_{k}} u(y)(y-x)^{-1} d y, \quad x \in \gamma_{j},
$$

где $u \in C_{0}^{\infty}(\Gamma \backslash z)$.

2. Узел, составленный из логарифмических спиралей. Проанализируем сначала структуру операторов $S_{j k}$ в каноническом случае, когда кривые $\gamma_{j}$ являются логарифмическими спиралями с началом в нуле и задаются уравнениями

$$
\begin{gathered}
\gamma_{j}=\left\{x \in \mathbb{C}: x=\varphi_{j}(s)=\right. \\
\left.\quad s \exp i\left(\delta \ln s+\theta_{j}\right), s \in \mathbb{R}_{+}\right\}, \quad j=1, \ldots, N, \quad \theta_{j}-\text { const } \\
0 \leqslant \theta_{1}<\theta_{2}<\cdots<\theta_{N}<2 \pi
\end{gathered}
$$

Сделав в (3.9) замену переменных $y=\varphi_{k}(\tau), x=\varphi_{j}(t), t, \tau \in \mathbb{R}_{+}$, получим, что оператор $S_{j k}$ перейдет в оператор меллиновской свертки

$$
\begin{aligned}
&\left(\widetilde{S}_{j k} v_{k}\right)(t)= \pm(\pi i)^{-1}(1+i \delta) \exp \left(i\left(\theta_{k}-\theta_{j}\right)\right) \\
& \times \int_{0}^{\infty}\left(\exp \left(i\left(\theta_{k}-\theta_{j}\right)\right)-\left(t \tau^{-1}\right)^{1+i \delta}\right)^{-1} v_{k}(\tau) \tau^{-1} d \tau \\
& t \in \mathbb{R}_{+}, \quad v_{k}(\tau) \in C_{0}^{\infty}\left(\mathbb{R}_{+}\right) .
\end{aligned}
$$


Оператор $\widetilde{S}_{j k}$ является оператором свертки на мультипликативной группе $\mathbb{R}_{+}$ с инвариантной мерой $d \mu=t^{-1} d t$ и действует в пространстве $L_{p, \beta+1 / p}\left(\mathbb{R}_{+}, d \mu\right)$, $\beta=\beta(z)$. Его будем обозначать $\widehat{S}_{j k}(\lambda)$, причем $\widehat{S}_{j k}(\lambda)$ с точностью до знака равен интегралу

$$
I(\lambda, \zeta, a)=(\pi i)^{-1} \zeta a \int_{0}^{\infty}(a-\exp (\zeta \ln t))^{-1} t^{-i \lambda-1} d t,
$$

где $a=\exp \left(i\left(\theta_{k}-\theta_{j}\right)\right), \quad \zeta=1+i \delta, \lambda \in \mathbb{R}_{\beta+1 / p}$. При $a=1$ интеграл (3.11) понимается в смысле главного значения.

ПРЕДЛОЖЕНИЕ 3.2. Пусть $0<\operatorname{Im} \lambda<1, \operatorname{Re} \zeta>\operatorname{Im} \lambda$. Тогда

$$
I(\lambda, \zeta, a)=2 a \exp \left[\left(-i \lambda \zeta^{-1}-1\right) \ln a\right]\left(\exp 2 \pi \lambda \zeta^{-1}-1\right)^{-1},
$$

если $a \in \mathbb{C} \backslash \overline{\mathbb{R}}_{+}$,

$$
I(\lambda, \zeta, 1)=\operatorname{cth}\left(\pi \lambda \zeta^{-1}\right) .
$$

ДокАЗАТЕЛЬСТво. Пусть $a \in \mathbb{C} \backslash \overline{\mathbb{R}}_{+} ;$тогда при условиях предложения 3.2 $I(\lambda, \zeta)$ есть аналитическая функция параметра $\zeta$ в области $\operatorname{Re} \zeta>\operatorname{Im} \lambda$. Найдем интеграл (3.11) в случае, когда $\operatorname{Im} \zeta=0$. Сделаем в (3.11) замену переменных $\tau=\exp (\zeta \ln t)=t^{\zeta} ;$ тогда

$$
I(\lambda, \zeta)=(\pi i)^{-1} a \int_{0}^{\infty}(a-\tau)^{-1} \tau^{-i(\lambda / \zeta)-1} d \tau .
$$

Интеграл (3.14) равен правой части в (3.12) при вешественных $\zeta>\operatorname{Im} \lambda$, и, следовательно, в силу принципа аналитического продолжения верна формула (3.12) в области $\operatorname{Re} \zeta>\operatorname{Im} \lambda$. Формула (3.14) получается из формулы (3.13) и предельных свойств особого интеграла типа Коши.

ПреДЛОЖЕНИЕ 3.3. Справедливы следующие формуль:

$$
\begin{gathered}
\widehat{S}_{j k}(\lambda)=\exp \left[\lambda \zeta^{-1}\left(\theta_{k}-\theta_{j}-\pi\right)\right]\left(\operatorname{sh} \pi\left(\lambda \zeta^{-1}\right)\right)^{-1}, \\
k>j, \quad \lambda \in \mathbb{R}_{\beta+1 / p}, \quad \zeta=1+i \delta, \\
\widehat{S}_{j k}(\lambda)=\exp \left[\lambda \zeta^{-1}\left(\theta_{k}-\theta_{j}+\pi\right)\right]\left(\operatorname{sh} \pi\left(\lambda \zeta^{-1}\right)\right)^{-1}, \\
k<j, \quad \lambda \in \mathbb{R}_{\beta+1 / p}, \quad \zeta=1+i \delta, \\
\widehat{S}_{j j}(\lambda)=\operatorname{cth}\left(\pi \lambda \zeta^{-1}\right), \quad \lambda \in \mathbb{R}_{\beta+1 / p}, \quad \zeta=1+i \delta .
\end{gathered}
$$

Это предложение следует из предложения 3.2 .

ЗАмечание. Пусть $\mathscr{L}_{\delta, \beta+1 / p}=\{\lambda=\nu+i \mu: \delta \nu+\mu=\beta+1 / p\}-$ прямая в комплексной плоскости; тогда формулы (3.15) можно переписать в виде

$$
\begin{aligned}
& \widehat{S}_{j k}(\lambda)=\exp \left[\lambda\left(\theta_{k}-\theta_{j}-\pi\right)\right](\operatorname{sh} \pi \lambda)^{-1}, \quad k>j, \quad \lambda \in \mathscr{L}_{\delta, \beta+1 / p}, \\
& \widehat{S}_{j k}(\lambda)=\exp \left[\lambda \zeta^{-1}\left(\theta_{k}-\theta_{j}+\pi\right)\right](\operatorname{sh} \pi \lambda)^{-1}, \quad k<j, \quad \lambda \in \mathscr{L}_{\delta, \beta+1 / p}, \\
& \widehat{S}_{j j}(\lambda)=\operatorname{cth} \pi \lambda, \quad \lambda \in \mathscr{L}_{\delta, \beta+1 / p} .
\end{aligned}
$$

Таким образом, формулы для символа СИО на системе логарифмических спиралей имеют близкий вид к формулам для СИО на системе лучей. Отличие заключается в том, что для системы лучей символ определен на прямой $\mathbb{R}_{\beta+1 / p}$, параллельной вешественной оси, а для системы логарифмических спиралей символ определен на прямой $\mathscr{L}_{\delta, \beta+1 / p}$ с угловым коэффициентом $k=-\delta$, проходящей через точку $(0, \beta+1 / p)$.

При $\delta=0$ формулы (3.16) переходят в известные формулы для символа СИО на системе лучей (см., например, [3], [7]). 
3. Общий случай. Рассмотрим теперь общий случай кривых вида (3.5), составляющих узел $z \in F$. Сделаем замену переменных $y=\varphi_{k}(\tau), x=\varphi_{j}(t)$; тогда оператор $S_{j k}$ перейдет в оператор

$$
\begin{gathered}
\left(\widetilde{S}_{j k} v_{k}\right)(t)= \pm(\pi i)^{-1} \chi_{j}(t) \int_{0}^{\infty} \chi_{k}(\tau) v_{k}(\tau) \varphi_{k}^{\prime}(\tau)\left(\varphi_{k}(\tau)-\varphi_{j}(t)\right)^{-1} d \tau, \quad t \in \mathbb{R}_{+} \\
v_{k}(\tau)=\left.u\right|_{\gamma_{k}}\left(z+\varphi_{k}(\tau)\right), \quad \chi_{k}(t)=\left.\chi\right|_{\gamma_{k}}\left(z+\varphi_{k}(\tau)\right),
\end{gathered}
$$

где $\chi(x) \in C_{0}^{\infty}(\mathbb{C})$ равна 1 в малой окрестности точки $z$ и имеет носитель в окрестности узла $z$. Знак " + " берется в том случае, когда контур $Г$ ориентирован так, что $\gamma_{k}$ выходит из узла $z$, а знак “ - ", когда $\gamma_{k}$ входит в узел $z$.

В силу оценки $\left|\varphi_{j}(t)\right| \geqslant t$, вытекающей из предложения 3.1 , пространство $L_{p}(\Gamma, \omega)$ локально изоморфно в окрестности узла $z$ пространству $L_{p, \beta}\left(\mathbb{R}_{+}, \mathbb{C}^{N}\right)$, $\beta=\beta(z)$, поэтому оператор $\widetilde{S}_{j k}$ будем рассматривать как оператор, действуюший в $L_{p, \beta}\left(\mathbb{R}_{+}\right)$. В случае $k=j$ интеграл в выражении для $\widetilde{S}_{j j}$ будем понимать в смысле главного значения.

Пусть $j \neq k$; тогда $\widetilde{S}_{j k}$ есть интегральньй оператор

$$
\left(\widetilde{S}_{j k} u\right)(t)=(\pi i)^{-1} \int_{0}^{\infty} k_{j k}(t, \tau) u(\tau) \tau^{-1} d \tau
$$

где

$$
\begin{gathered}
k_{j k}(t, \tau)= \pm \chi_{j}(t) \chi_{k}(\tau) a_{k j}(t, \tau)\left(1+i \delta+i \tau \theta_{k}^{\prime}(\tau)\right)\left(a_{k j}(t, \tau)-\left(t \tau^{-1}\right)^{1+i \delta}\right)^{-1} \\
a_{k j}(t, \tau)=\exp i\left(\theta_{k}(t)-\theta_{j}(\tau)\right) .
\end{gathered}
$$

Используя формулу (3.12), запишем оператор $\widetilde{S}_{j k}$ формально как ПДО Меллина

$$
\left(\widetilde{S}_{j k} u\right)(t)=(2 \pi)^{-1} \iint_{\mathbb{R}_{+} \times \mathbb{R}_{\beta+1 / p}} s_{j k}(t, \tau, \lambda)\left(t \tau^{-1}\right)^{i \lambda} u(\tau) \tau^{-1} d \tau d \lambda
$$

с двойньм символом

$$
\begin{aligned}
s_{j k}(t, \tau, \lambda)= & \pm 2 a_{k j}(t, \tau)\left[\left(1+i \tau \zeta^{-1} \theta_{k}^{\prime}(\tau)\right)\right] \chi_{j}(t) \chi_{k}(\tau) \\
& \times \exp \left[\left(-i \lambda \zeta^{-1}-1\right) \ln a_{k j}(t, \tau)\right]\left[\exp \left(2 \pi \lambda \zeta^{-1}-1\right)\right]^{-1}, \quad \zeta=1+i \delta .
\end{aligned}
$$

Из условия (3.6) следует, что

$$
\begin{aligned}
& \ln a_{k j}(t, \tau)=i\left(\theta_{k}(t)-\theta_{j}(\tau)\right), \quad k>j, \\
& \ln a_{k j}(t, \tau)=i\left(2 \pi-\left(\theta_{k}(t)-\theta_{j}(\tau)\right)\right), \quad k<j .
\end{aligned}
$$

Несложная проверка, используюшая условия (3.1) и (3.6), показывает, что $s_{j k}(t, \tau, \lambda) \quad(j \neq k)$ удовлетворяет условиям определения 2.3 , т.е. $\widetilde{S}_{j k}$ есть ПДО Меллина с двойным символом, медленно меняюшимся в точке нуль.

Заметим, что для любых целых неотрицательных $\alpha, \beta, \gamma$

$$
\lim _{\lambda \rightarrow \infty} \sup _{\rho, r \in \mathbb{R}_{+}}\left|\left(r \partial_{r}\right)^{\beta}\left(\rho \partial_{\rho}\right)^{\gamma} \partial^{\alpha} s_{j k}(r, \rho r, \lambda)\right|\langle\lambda\rangle^{\alpha}=0 .
$$


Пусть теперь $j=k$; тогда

$$
\left(\widetilde{S}_{j j} u\right)(t)=(\pi i)^{-1} \int_{0}^{\infty} k_{j j}(t, \tau) u(\tau) \tau^{-1} d \tau
$$

где

$$
\begin{aligned}
k_{j j}(t, \tau) & = \pm \chi_{j}(t) \chi_{j}(\tau)\left(1+i \delta+i \tau \theta_{k}^{\prime}(\tau)\right) b_{j}^{-1}(t, \tau)\left[1-\left(t \tau^{-1}\right)^{1+i \delta}\right]^{-1} \\
b_{j}(t, \tau) & =\exp \left(i \theta_{j}(\tau)\right)+\frac{\left(\exp \left(i \theta_{j}(\tau)\right)-\exp \left(i \theta_{j}(t)\right)\right) t^{1+i \delta}}{\tau^{1+i \delta}-t^{1+i \delta}}
\end{aligned}
$$

Из предложения 3.1 следует, что сушествуют такие константы $c, C>0$, что $c<\left|b_{j}(t, \tau)\right|<C$ для достаточно малых $t, \tau$.

Рассмотрим теперь второе слагаемое в правой части формулы (3.19), которое мы обозначим через $d_{j}(t, \tau)$. Воспользовавшись теоремой о среднем, запишем $d_{j}(t, \tau)$ в виде

$$
d_{j}(t, \tau)=\frac{t^{1+i \delta} \int_{0}^{1} i \theta^{\prime}(t s+(1-s) \tau) \exp i \theta(t s+(1-s) \tau) d s}{\int_{0}^{1}(1+i \delta)(t s+(1-s) \tau)^{i \delta} d s}=\frac{d_{j 1}(t, \tau)}{d_{j 2}(t, \tau)}
$$

Покажем, что $\lim _{t \rightarrow+0} d_{j}(t, t \tau)=0$ равномерно по $\tau \in[\varepsilon, 1 / \varepsilon]$ для любого $\varepsilon>0$. Действительно, $s+(1-s) \tau \geqslant \tau \geqslant \varepsilon$, если $0<\tau \leqslant 1$, и $s+(1-s) \tau \geqslant 1$, если $\tau \geqslant 1$. Таким образом, если $M=\max \{1 / \varepsilon, 1\}$, то

$$
\left|d_{j 1}(t, t \tau)\right| \leqslant M|t(s+(1-s) \tau)|\left|\theta^{\prime}(t(s+(1-s) \tau))\right|,
$$

следовательно, $\lim _{t \rightarrow+0} d_{j 1}(t, t \tau)=0$ равномерно по $s \in[0,1], \tau \in[\varepsilon, 1 / \varepsilon]$ в силу условий (3.1). Из предложения 3.1 следует, что $\inf _{t, \tau \in \mathbb{R}_{+}}\left|d_{2 j}(t, \tau)\right| \geqslant c>0$, следовательно, сформулированное вьше утверждение справедливо.

Нетрудно проверить, что для любых $\alpha, \beta$

$$
\left|(t d / d t)^{\alpha}(\tau d / d \tau)^{\beta} d_{j}(t, t \tau)\right| \leqslant C_{\alpha \beta}
$$

когда $t$ и $t \tau$ меняются в малой окрестности нуля, более того, для любых $\alpha, \beta$

$$
\lim _{t \rightarrow+0}(t d / d t)^{\alpha}(\tau d / d \tau)^{\beta} d_{j}(t, t \tau)=0
$$

равномерно по $\tau \in[\varepsilon, 1 / \varepsilon]$.

Запишем теперь $\widetilde{S}_{j j}$ в виде ПДО Меллина $(3.18)$ с двойным символом

$$
s_{j j}(t, \tau, \lambda)= \pm \chi_{j}(t) \chi_{j}(\tau)\left(1+i \delta+i \tau \theta_{k}^{\prime}(\tau)\right) b_{j}^{-1}(t, \tau)(1+i \delta)^{-1} \operatorname{cth} \pi(1+i \delta)^{-1} \lambda .
$$

Из (3.20) и (3.21) следует, что $s_{j j}(t, \tau, \lambda)$ удовлетворяет оценкам (2.3) и медленно меняется в нуле. 
Из предложения 2.1 теперь следует

ПРЕДЛОЖЕНИЕ 3.4. Матричный оператор $\widetilde{S}_{z}=\left(\widetilde{S}_{j k}\right)_{j, k=1}^{N} \in O P \mathscr{E}_{\beta+1 / p}(N)$, $N=N(z), \beta=\beta(z)$. Его локальныи символ в точке нуль есть

$$
\Omega^{z}(t, \lambda)=\left(\Omega_{j k}^{z}(t, \lambda)\right)_{j, k=1}^{N(z)}
$$

где $\Omega_{j k}^{z}(t, \lambda)$ виччисляются по формулам

$$
\begin{aligned}
& \Omega_{j k}^{z}(t, \lambda)= \pm \exp \left[\lambda \zeta^{-1}\left(\theta_{k}(t)-\theta_{j}(t)-\pi\right)\right]\left(\operatorname{sh} \pi\left(\lambda \zeta^{-1}\right)\right)^{-1}, \quad k>j, \quad \lambda \in \mathbb{R}_{\beta+1 / p}, \\
& \Omega_{j k}^{z}(t, \lambda)= \pm \exp \left[\lambda \zeta^{-1}\left(\theta_{k}(t)-\theta_{j}(t)+\pi\right)\right]\left(\operatorname{sh} \pi\left(\lambda \zeta^{-1}\right)\right)^{-1}, \quad k<j, \quad \lambda \in \mathbb{R}_{\beta+1 / p}, \\
& \Omega_{j j}(\lambda)= \pm \operatorname{cth}\left(\pi \lambda \zeta^{-1}\right), \quad \zeta=1+i \delta .
\end{aligned}
$$

Здесь $t \in(0, s)$, әде s достаточно мало, $\lambda \in \mathbb{R}_{\beta+1 / p}$, знак "+" берется, если контур Г ориентирован так, что кривая $\gamma_{k}$ выходит из узла, и знак “-”, если кривая $\gamma_{k}$ входит в узел.

Положим

$$
\sigma_{\Gamma}^{z}(t, \mu)=\Omega^{z}(t, \mu \zeta), \quad \mu \in \mathscr{L}_{\delta, \beta+1 / p}
$$

Тогда формулы для локального символа СИО становятся похожими на формулы для локального символа СИО на системе лучей [3], [7] с тем отличием, что $\theta_{j}=\theta_{j}(t)$ и параметр $\mu$ меняется на прямой $\mathscr{L}_{\delta, \beta+1 / p}$ в комплексной плоскости, имеющей угловой коэффициент $k=-\delta$.

Сопоставим теперь СИО $S_{\Gamma}$ в точке $x \in \Gamma / F$, как обычно, символ $\sigma_{\Gamma}^{x}(\xi)=\operatorname{sgn} \xi$, $\xi \in \mathbb{R}$. Пусть теперь $a(x) \in P S V(\Gamma, F) ;$ сопоставим этой функции в узле $z \in F$ оператор умножения на диагональную матрищу

$$
a^{z}(t)=\operatorname{diag}\left(a_{1}^{z}(t), \ldots, a_{N(z)}^{z}(t)\right),
$$

где $a_{k}^{z}(t)=\left.a\right|_{\gamma_{k}}\left(z+\varphi_{k}(\tau)\right) \in S V\left(\mathbb{R}_{+}\right)$, действуюший в $L_{p, \beta}\left(\mathbb{R}_{+}, \mathbb{C}^{N(z)}\right)$.

ОПРЕДЕЛЕНИЕ 3.3. Обозначим через $\mathfrak{M}_{p}(\Gamma, \omega)$ банахову алгебру операторов, действующих в $L_{p}(\Gamma, \omega)$, порожденную операторами вида

$$
A=\sum_{k} \prod_{l} A^{k l}
$$

где $A^{k l}$ - либо СИО $S_{\Gamma}$, либо оператор умножения на функцию $a(x) \in P S V(\Gamma, F)$, $k, l$ пробегают конечное множество индексов.

Можно показать (см., например, [3], [6] ), что $\mathfrak{M}_{p}(\Gamma, \omega)$ неприводима и содержит двухсторонний идеал $\mathfrak{K}\left(L_{p}(\Gamma, \omega)\right)$. Операторы из алгебры $\mathfrak{M}_{p}(\Gamma, \omega)$ локального типа на $Г$.

Положим

$$
\sigma^{x}(A)=\sum_{p} \prod_{q} \sigma^{x}\left(A^{k l}\right),
$$

где: $\sigma^{x}\left(A^{k l}\right)=\sigma_{\Gamma}^{x}(t, \mu), t \in\left(0, s_{x}\right), \mu \in \mathscr{L}_{\delta, \beta(x)+1 / p}$, если $A^{k l}=S_{\Gamma}$ и $x \in F$; $\sigma^{x}\left(A^{k l}\right)=\operatorname{sgn} \xi$, если $x \in \Gamma \backslash F$ и $A^{k l}=S_{\Gamma} ; \sigma^{x}\left(A^{k l}\right)$ есть оператор умножения на $a^{x}(t)$, если $A^{k l}$ - оператор умножения на $a(x) \in P S V(\Gamma, F)$ и $x \in F ; \sigma^{x}\left(A^{k l}\right)=$ $a(x)$, если $A^{k l}$ - оператор умножения на $a(x) \in P S V(\Gamma, F)$.

Таким образом, $\sigma^{x}(A) \in \mathscr{R}_{p, \beta(x)}(N(x))$, если $x \in F$, и $\sigma^{x}(A)=\left(\sigma_{+}^{x}(A)\right.$, $\left.\sigma_{-}^{x}(A)\right) \in \mathbb{C} \oplus \mathbb{C}$, если $x \in \Gamma / F$. 
TeOpema 3.1. Oператор А вида (3.22) локально обратим:

(a) в точке $x \in \Gamma / F$ тогда и только тогда, когда

$$
\sigma_{ \pm}^{x}(A) \neq 0
$$

(b) в точке $x \in F$ тогда и только тогда, когда

$$
\lim _{s \rightarrow 0} \inf _{t \in(0, s), \lambda \in \mathscr{L}_{\delta, \beta(x)+1 / p}}\left|\operatorname{det} \sigma^{x}(A)(t, \lambda)\right|>0 .
$$

ЗАмЕчАниЕ. Условие (3.25) равносильно условию обратимости факторкласса $\hat{\sigma}^{x}(A)$ в $\mathscr{R}_{p, \beta(x)}^{+}(N(x))$.

Утверждение теоремы 3.1 для точек $x \in \Gamma / F$ хорошо известно (см. [1], [5]), а для точек $x \in F$ оно следует из теоремы 2.1.

Теорема 3.2. Пусть A- оператор вида (3.21). Тогда следующие условия равносильны:

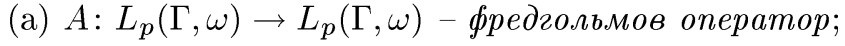

(b) $\forall x \in \Gamma / F$ выполнено условие (3.24), а в любой точке $x \in F$ выполнено условие (3.25).

Теорема 3.2 следует стандартным образом из локального принципа и теоремы 3.1 .

Через $\mathcal{T}_{p, z}(\Gamma, \omega)$ обозначим двухсторонний идеал в $\mathfrak{M}_{p}(\Gamma, \omega)$, полученный замыканием в $\mathfrak{L}\left(L_{p}(\Gamma, \omega)\right)$ множества операторов вида (3.20), где $\forall k \exists l$ такое, что $A^{k l}$ есть оператор умножения на такую функцию $a(x) \in P S V(\Gamma, F)$, что $\lim _{\Gamma \in x \rightarrow z} a(x)=0$.

TeOpema 3.3. Пусть A - оператор вида (3.22). Тогда

$$
\begin{gathered}
\left\|\hat{\sigma}^{x}(A)\right\| \leqslant\|A\|\left\|_{x}=\inf _{T \in \mathcal{T}_{x}(\Gamma, \omega)}\right\| A-T\|\leqslant c\| \hat{\sigma}^{x}(A) \|, \\
\text { əде }\left\|\hat{\sigma}^{x}(A)\right\|=\max \left(\left|\sigma_{+}^{x}(A)\right|,\left|\sigma_{-}^{x}(A)\right|\right), \quad \text { ecли } \quad x \in \Gamma / F, \quad \text { u } \quad\left\|\hat{\sigma}^{x}(A)\right\|= \\
\left\|\hat{\sigma}^{x}(A)\right\|_{\mathscr{R}_{p, \beta(x)}^{+}}, \text {если } x \in F .
\end{gathered}
$$

Доказательство теоремы 3.3 заменой переменных сводится к теореме 2.2.

Teоpema 3.4. Пусть A - оператор вида (3.22) $и$

$$
\left\||\|A\||=\inf _{T \in \mathfrak{K}\left(L_{p}(\Gamma, \omega)\right)}\right\| A-T \|_{\mathfrak{L}\left(L_{p}(\Gamma, \omega)\right)}
$$

Тогда

$$
\sup _{x \in \Gamma}\left\|\hat{\sigma}_{x}(A)\right\| \leqslant\|\| A\|\| \leqslant \sup _{x \in \Gamma}\left\|\hat{\sigma}_{x}(A)\right\| .
$$

ДокАЗАТЕльСтво. Оценка снизу в (3.25) следует из теоремы 3.3, а оценка сверху - из теоремы об огибающей [5].

Теоремы $3.3,3.4$ и предельный переход позволяют определить локальный $\hat{\sigma}_{x}(A)$, $x \in \Gamma$, и глобальный $\sigma(A)=\left\{\hat{\sigma}_{x}(A)\right\}_{x \in \Gamma}$ символы оператора $A \in \mathfrak{M}_{p}(\Gamma, \omega)$. 
ОПРЕДЕЛЕНИЕ 3.4 . Через $\mathfrak{N}_{p}(\Gamma, \omega)$ будем обозначать банахову алгебру символов $\sigma(A)$ операторов из $\mathfrak{M}_{p}(\Gamma, \omega)$ с поточечными операциями и нормой

$$
\|\sigma(A)\|=\sup _{x \in \Gamma}\left\|\hat{\sigma}_{x}(A)\right\| .
$$

Teорема 3.5. (а) Отображсение $\Sigma_{p}: \mathfrak{M}_{p}(\Gamma, \omega) \rightarrow \mathfrak{N}_{p}(\Gamma, \omega)$, сопоставляющее оператору его символ, есть мономорфизм банаховых алгебр $\mathfrak{M}_{p}(\Gamma, \omega)$ и $\mathfrak{N}_{p}(\Gamma, \omega)$, ядром которого является двухсторонний идеал $\mathfrak{K}\left(L_{p}(\Gamma, \omega)\right)$.

(b) $A \in \mathfrak{M}_{p}(\Gamma, \omega)$ фредгольмов в $L_{p}(\Gamma, \omega)$ тогда и только тогда, когда его символ обратим в $\mathfrak{N}_{p}(\Gamma, \omega)$, что равносильно тому, что $\sigma_{x}^{ \pm}(A) \neq 0$, когда $x \in \Gamma \backslash F$, и выполнено условие (3.25), когда $x \in F$.

(c) Если $A \in \mathfrak{M}_{p}(\Gamma, \omega)$ фредгольмов в $L_{p}(\Gamma, \omega)$, то его регуляризатор $R$ принадлежсат алгебре $\mathfrak{M}_{p}(\Gamma, \omega)$ и $\sigma(R)=\sigma(A)^{-1}$.

Теорема 3.5 вытекает из локального принципа и теоремы 3.2 .

4. Банахова алгебра $\mathfrak{N}_{p}(\Gamma, \omega)$. Пусть $\theta(t)$ - бесконечно дифференцируемая на $\mathbb{R}_{+}$вещественная функция, равная нулю вне некоторой окрестности точки нуль и такая, что $\forall k \in \mathbb{N}$

$$
\lim _{t \rightarrow+0}(t \partial / \partial t)^{k} \theta(t)=0 .
$$

Пусть $0<\theta_{1} \leqslant \theta(t) \leqslant \theta_{2}<2 \pi ;$ положим

$$
\begin{aligned}
\left(N_{\delta, \theta(t)} f\right)(t) & =(\pi i)^{-1}(1+i \delta) \int_{\mathbb{R}_{+}} f(s)\left(s^{1+i \delta}-(\exp i \theta(t)) t^{1+i \delta}\right)^{-1} s^{i \delta} d s, \quad t \in \mathbb{R}_{+}, \\
\left(S_{\delta,+} f\right)(t) & =(\pi i)^{-1}(1+i \delta) \int_{\mathbb{R}_{+}} f(s)\left(s^{1+i \delta}-t^{1+i \delta}\right)^{-1} s^{i \delta} d s, \quad t \in \mathbb{R}_{+} .
\end{aligned}
$$

Операторы $N_{\delta, \theta(t)}, S_{\delta,+} \in O P \widetilde{\mathscr{E}}_{\beta+1 / p}$ и их символы есть соответственно

$$
\begin{gathered}
\exp \lambda \zeta^{-1}(\theta(t)-\pi) / \operatorname{sh} \pi \lambda \zeta^{-1}, \quad \lambda \in \mathbb{R}_{\beta+1 / p}, \quad \zeta=1+i \delta \\
\operatorname{cth} \pi \lambda \zeta^{-1}, \quad \lambda \in \mathbb{R}_{\beta+1 / p}, \quad \zeta=1+i \delta .
\end{gathered}
$$

Заметим, что $N_{\delta, \theta(t)} N_{\delta, 2 \pi-\theta(t)}=S_{\delta,+}^{2}-I+T$, где $T \in \mathscr{R}_{p, \beta}^{0}$.

Через $\Omega(p, \beta, \delta)$ обозначим банахову алгебру операторов, действуюших в $L_{p, \beta}\left(\mathbb{R}_{+}\right),-1 / p<\beta<1-1 / p$, порожденную оператором $S_{\delta,+}$, всеми операторами $N_{\delta, \theta(t)}$, где $\theta(t)$ удовлетворяют оценкам (3.27), и операторами умножения на функции из $S V\left(\mathbb{R}_{+}\right)$, а через $W(p, \beta, \delta)$ соответственно банахову алгебру операторов, действуюших в $L_{p, \beta}\left(\mathbb{R}_{+}\right)$, порожденную всеми операторами $N_{\delta, \theta(t)}$, где $\theta(t)$ удовлетворяет оценкам $(3.27)$, и операторами умножения на функции из $S V\left(\mathbb{R}_{+}\right)$.

Заметим, что $\Omega(p, \beta, \delta), W(p, \beta, \delta)$ являются подалгебрами банаховой алгебры $\mathscr{R}_{p, \beta}=\mathscr{R}_{p, \beta}(1)$ для любого $\delta \in \mathbb{R}$ и $W(p, \beta, \delta)$ - двухсторонний идеал в $\Omega(p, \beta, \delta)$.

Пусть $\mathfrak{D}^{N}(p, \beta, \delta) \subset \mathscr{R}_{p, \beta}(N)$ - банахова алгебра матричных операторов $A=\left(A_{i j}\right)_{i, j=1}^{N}$, где $A_{i i} \in \Omega(p, \beta, \delta), A_{i j} \in W(p, \beta, \delta), i \neq j$, a $\widetilde{\mathfrak{D}}^{N}(p, \beta, \delta)$ - coответствующая алгебра локальных в точке нуль матричных символов. Положим $\mathcal{T}^{N}(p, \beta, \delta)=\mathfrak{D}^{N}(p, \beta, \delta) \cap \mathscr{R}_{p, \beta}^{0}(N)$. 
ПРеДЛОЖЕНИЕ 3.5. Пусть $x \in F$, факторалгебра $\mathfrak{M}_{p}(\Gamma, \omega) / \mathcal{T}_{p, x}(\Gamma, \omega)$ изометрически изоморфна факторалгебре

$$
\mathfrak{D}^{N(x)}(p, \beta(x), \delta(x)) / \mathcal{T}^{N(x)}(p, \beta(x), \delta(x)) .
$$

Изоморфизм порождается отображением, сопоставляющим оператору $A \in \mathfrak{M}_{p}(\Gamma, \omega)$ его локальный операторный символ в точке $x \in F$.

Предложение 3.5 следует из теоремы 3.3 .

Отметим, что локальные матричные символы $\hat{a} \in \widetilde{\mathfrak{D}}^{N}(p, \beta, \delta)$ таковы, что если $a=\left(a_{i j}(t, \lambda)\right)_{i, j=1}^{N}\left(t \in \mathbb{R}, \lambda \in \mathscr{L}_{\delta, \beta+1 / p}\right)$ - представитель факторкласса $\hat{a}$, то существуют пределы

$$
\begin{aligned}
& \lim _{\nu \rightarrow \pm \infty} a_{i i}(t, \nu+i(-\delta \nu+\beta+1 / p))=a_{i i}(t, \pm), \\
& \lim _{\nu \rightarrow \pm \infty} a_{i j}(t, \nu+i(-\delta \nu+\beta+1 / p))=0, \quad i \neq j .
\end{aligned}
$$

Обозначим через $\mathfrak{A}_{p}(\Gamma, \omega)$ множество, элементами которого являются семейства $a=\left\{a_{x}\right\}_{x \in \Gamma}$, где $a_{x}=\left(a_{x}^{ \pm}\right)$, если $x \in \Gamma / F$ и $a_{x}^{ \pm}$- непрерывные функции на $\Gamma \backslash F$, и $a_{x}=\left(a_{x}^{i j}(t, \lambda)\right)_{i, j=1}^{N(x)} \in \widetilde{\mathfrak{D}}^{N(x)}(p, \beta(x), \delta(x))$, если $x \in F$.

Положим

$$
a_{x}^{i i}(t, \pm)=\lim _{\nu \rightarrow \pm \infty} a_{x}^{i i}(t, \nu+i(-\delta \nu+\beta+1 / p)) .
$$

Между $a_{x}$ в регулярных точках $x \in \Gamma \backslash F$ и узлах $x \in F$ устанавливается следующая связь.

Пусть $x \in F$ и $\gamma_{i}(x)(i=1, \ldots, N(x))$ - кривые, концами которых служит точка $x, z=x+\varphi_{i}(t)$ - их параметризация в окрестности конца $x \in F$; тогда будем предполагать, что

$$
\lim _{t \rightarrow+0}\left(a_{x+\varphi_{i}(t)}^{ \pm}-a_{x}^{i i}(t, \pm)\right)=0, \quad i=1, \ldots, N(x),
$$

если контур Г ориентирован так, что $\gamma_{i}(x)$ выходит из узла $x$, и

$$
\lim _{t \rightarrow+0}\left(a_{x+\varphi_{i}(t)}^{ \pm}-a_{x}^{i i}(t, \mp)\right)=0, \quad i=1, \ldots, N(x),
$$

если контур $Г$ ориентирован так, что $\gamma_{i}(x)$ входит в узел $x$.

Введем естественные поточечные операции сложения, умножения, инволюции во множестве $\mathfrak{A}_{p}(\Gamma, \omega)$ и норму, как и в определении 3.4 ; тогда $\mathfrak{A}_{p}(\Gamma, \omega)$ превращается в банахову алгебру.

Теорема 3.6. Банаховы алгебры $\mathfrak{N}_{p}(\Gamma, \omega)$ и $\mathfrak{A}_{p}(\Gamma, \omega)$ изометрически изоморфны.

Доказательство теоремы 3.6 основано на тех же соображениях, что и доказательство аналогичного результата для банаховой алгебры, порожденной СИО с кусочно-непрерывными коэффициентами на кусочно-ляпуновских контурах (см. [3], [5]), и проводится следуюшим образом. Легко проверить, что $\mathfrak{N}_{p}(\Gamma, \omega)$ естественно вложена в $\mathfrak{A}_{p}(\Gamma, \omega)$. Более того, это вложение есть эпиморфизм. Действительно, если $a=\left(a_{x}\right)_{x \in \Gamma} \in \mathfrak{A}_{p}(\Gamma, \omega)$, то для любого $x \in \Gamma$ найдется оператор $A_{x} \in \mathfrak{M}_{p}(\Gamma, \omega)$ такой, что $\sigma_{x}\left(A_{x}\right)=a_{x}$. Из непрерьвности семейств $a_{x}^{ \pm}$в точках $\Gamma / F$ и условий $(3.28),(3.29)$ следует, что семейство операторов $\left\{A_{x}\right\}_{x \in \Gamma}$ непрерывно в точках $\Gamma / F$ и локально полунепрерывно в каждой точке $x \in F$. В силу теоремы об огибающей (см. [5, с. 26]) существует оператор $A \in \mathfrak{M}_{p}(\Gamma, \omega)$, локально эквивалентный в каждой точке контура $\Gamma$ оператору $A_{x}$, где $\sigma_{x}\left(A_{x}\right)=a_{x}$. Таким образом, $\sigma(A)=\left(a_{x}\right)_{x \in \Gamma}$, т.е. $\sigma(A) \in \mathfrak{N}_{p}(\Gamma, \omega)$. 


\section{$\S 4$. Сингулярные интегральные операторы на замкнутом контуре с точками завихрения}

Пусть $\Gamma$ - замкнутый контур на комплексной плоскости $\mathbb{C}$, разделяющий ее на две связные компоненты: ограниченную $D^{+}$и неограниченную $D^{-}$. Контур Г ориентирован таким образом, что при его положительном обходе $D^{+}$остается слева. Будем предполагать, что $\Gamma=\bigcup_{j=1}^{L} \gamma_{j}$, где $\gamma_{j} \in \mathfrak{R}_{0}$ и могут пересекаться только в конщевых точках. Множество таких точек, как и выше, обозначим через $F$.

Пусть $x=x_{j}+\varphi_{j}^{ \pm}(t), t \in\left(0, s_{j}\right),-$ параметризация контура Г в окрестности узла $x_{j}$, где $\varphi_{j}^{ \pm}(t)=t \exp i\left(\delta_{j} \ln t+\varkappa_{j}(t)+\theta_{j}^{ \pm}(t)\right)$, а $\varkappa_{j}(t), \theta_{j}^{ \pm}(t)$ удовлетворяют условиям (3.1) в окрестности точки нуль. Будем считать, что $x_{j}+\varphi_{j}^{ \pm}(t)$ лежит соответственно в правой (левой) окрестности узла $x_{j}$. Положим $\alpha_{j}(t)=\theta_{j}^{+}(t)-$ $\theta_{j}^{-}(t)$ и будем считать, что $0<\alpha_{j 0} \leqslant \alpha_{j}(t) \leqslant \alpha_{j 1}<2 \pi, t \in\left(0, s_{j}\right)$.

Как и в предыдущем параграфе, вводятся пространства $L_{p}(\Gamma, \omega)$, где $1<p<$ $\infty, \omega(x)=\prod_{j=1}^{L}\left|x-x_{j}\right|^{\beta_{j}}, \beta_{j} \in(-1 / p, 1-1 / p), x_{j} \in F$.

Через $P_{\Gamma}^{+}=\frac{1}{2}\left(I+S_{\Gamma}\right)$ и $P_{\Gamma}^{-}=\frac{1}{2}\left(I-S_{\Gamma}\right)$ будем, как обычно, обозначать сингулярные проекторы в пространстве $L_{p}(\Gamma, \omega)$. Положим

$$
A=a_{+}(x) P_{\Gamma}^{+}+a_{-}(x) P_{\Gamma}^{-}, \quad a_{+}(x), a_{-}(x) \in P S V(\Gamma, F) .
$$

Согласно формулам предыдушего параграфа символ $\sigma(A)$ оператора $A$ определяется следуюшим образом:

$1)$ если $x \in \Gamma \backslash F$, то $\sigma_{x}(A)$ есть пара числе $\left(a_{+}(x), a_{-}(x)\right)$;

2) если $x=x_{j} \in F$, то

$$
\begin{aligned}
\sigma_{x_{j}}\left(a_{ \pm}\right)(t) & =\left(\begin{array}{cc}
a_{ \pm}\left(x_{j}+\varphi_{j}^{+}(t)\right) & 0 \\
0 & a_{ \pm}\left(x_{j}+\varphi_{j}^{-}(t)\right)
\end{array}\right), \\
\sigma_{x_{j}}\left(P_{\Gamma}^{ \pm}\right)(t, \lambda) & =\frac{1}{2}\left(\begin{array}{ccc}
\left(1 \pm \operatorname{cth} \pi \Lambda_{j}\right) & \mp \exp \left[\Lambda_{j}\left(\alpha_{j}(t)-\pi\right)\right] / \operatorname{sh} \pi \Lambda_{j} \\
\pm \exp \left[\Lambda_{j}\left(\pi-\alpha_{j}(t)\right)\right] / \operatorname{sh} \pi \Lambda_{j} & \left(1 \pm \operatorname{cth} \pi \Lambda_{j}\right)
\end{array}\right),
\end{aligned}
$$

где $\Lambda_{j}=\lambda+i\left(-\delta \lambda+\beta_{j}+1 / p\right), \lambda \in \mathbb{R}$,

$$
\sigma_{x_{j}}(A)(t, \lambda)=\sigma_{x_{j}}\left(a_{+}\right)(t) \sigma_{x_{j}}\left(P_{\Gamma}^{+}\right)(t, \lambda)+\sigma_{x_{j}}\left(a_{-}\right)(t) \sigma_{x_{j}}\left(P_{\Gamma}^{-}\right)(t, \lambda),
$$

где $t$ меняется в достаточно малой окрестности нуля.

Tеорема 4.1. Onератор А фредгольмов в $L_{p}(\Gamma, \omega)$ тогда и только тогда, когда:

(a) $\inf _{\Gamma}\left|a_{+}(x)\right|>0, \inf _{\Gamma}\left|a_{-}(x)\right|>0$

(b) для любого узла $x_{j} \in F, j=1, \ldots, L$,

$$
\lim _{\varepsilon \rightarrow 0} \inf _{(t, \lambda) \in(0, \varepsilon) \times \mathbb{R}}\left|\operatorname{det} \sigma_{x_{j}}(A)(t, \lambda)\right|>0 .
$$

При условиях (a), (b) индекс оператора А вычисляется по формуле

$$
\begin{aligned}
\operatorname{Ind} A=- & \sum_{j=1}^{L}(2 \pi)^{-1}\left[\arg a_{+}(x) a_{-}^{-1}(x)\right]_{\gamma_{j}\left(z_{j}^{\prime}, z_{j}^{\prime \prime}\right)} \\
& \quad-\sum_{j=1}^{L}(2 \pi)^{-1}\left[\arg \operatorname{det} \sigma_{x_{j}}(A)\left(t_{j}, \lambda\right)\right]_{\lambda=-\infty}^{\infty},
\end{aligned}
$$


где $\gamma_{j}\left(z_{j}^{\prime}, z_{j}^{\prime \prime}\right)$ - часть дуги $\gamma_{j}$, лежащая между точками $z_{j}^{\prime}, z_{j}^{\prime \prime}, t_{j}$ - такая произвольная точка, принадлежащая $\mathbb{R}_{+}$, что

$$
\inf _{\lambda \in \mathbb{R}}\left|\operatorname{det} \sigma_{x_{j}}(A)(t, \lambda)\right|>0, \quad j=1, \ldots, L, \quad \forall t \in\left(0, t_{j}\right],
$$

а точки $z_{j}^{\prime}, z_{j}^{\prime \prime}$ определяются формулами

$$
\begin{gathered}
z_{1}^{\prime}=x_{1}+\varphi_{1}^{+}\left(t_{1}\right), \quad z_{1}^{\prime \prime}=x_{2}+\varphi_{2}^{-}\left(t_{2}\right), \quad z_{2}^{\prime}=x_{2}+\varphi_{2}^{+}\left(t_{2}\right), \\
z_{2}^{\prime \prime}=x_{3}+\varphi_{3}^{-}\left(t_{3}\right), \quad \ldots, \quad z_{L}^{\prime}=x_{L}+\varphi_{L}^{+}\left(t_{L}\right), \quad z_{L}^{\prime \prime}=x_{1}+\varphi_{1}^{-}\left(t_{1}\right) .
\end{gathered}
$$

ДокАЗАТЕЛЬСТво. Необходимые и достаточные условия фредгольмовости (а), (b) оператора $A$ вытекают из теоремы 3.2 .

Докажем формулу индекса оператора $A$. Для ее доказательства воспользуемся хорошо известным методом разделения особенностей. Выберем малые окрестности $\Gamma_{j}^{\prime \prime} \subset \Gamma_{j} \subset \Gamma_{j}^{\prime}$ узла $x_{j}, j=1, \ldots, L$, где каждая включает внутренность следующей и они не пересекаются, $\Gamma_{j}^{\prime} \cap \Gamma_{k}^{\prime}=\varnothing, j \neq k$. Пусть $\Gamma_{0}=\Gamma \backslash \bigcup_{j=1}^{L} \Gamma_{j}^{\prime}$, $\Gamma_{0}^{\prime}=\Gamma \backslash \bigcup_{j=1}^{L} \Gamma_{j}$. Очевидно, $\Gamma_{0} \subset \Gamma_{0}^{\prime}$ и оба семейства $\left\{\Gamma_{j}\right\}_{0}^{N},\left\{\Gamma_{j}^{\prime}\right\}_{0}^{N}$ покрывают $\Gamma$.

Пусть $\left\{\chi_{j}\right\}_{0}^{L}$ - гладкое разбиение единицы, подчиненное покрытию $\left\{\Gamma_{j}^{\prime}\right\}_{0}^{N}$ : $\operatorname{supp} \chi_{j} \subset \Gamma_{j}^{\prime}, \quad \sum_{0}^{L} \chi_{j}(x)=1, t \in \Gamma$ и $\chi_{j}(x)=1$ для $x \in \Gamma_{j}, j=0,1, \ldots, L$, $\chi_{j}(x) \chi_{k}(x)=0$ при $j \neq k(j, k=2, \ldots, L), \prod_{1}^{L}\left(1-\chi_{j}(x)\right)=\chi_{0}(x)$.

Согласно условию (а) сушествуют обратимые функции $a_{0}^{ \pm}(x)$ такие, что $a_{0}^{ \pm}(x)=a^{ \pm}(x)$, когда $x \in \Gamma_{0}^{\prime}$, и $a_{0}^{ \pm}(x)=1$, когда $x \in \bigcup_{j=1}^{L} \Gamma_{j}^{\prime \prime}$.

Положим

$$
A_{0}=a_{0}^{+}(x) P_{\Gamma}^{+}+a_{0}^{-}(x) P_{\Gamma}^{-}, \quad A_{j}=b_{0 j}^{+}(x) P_{\Gamma}^{+}+b_{0 j}^{-}(x) P_{\Gamma}^{-}+\left(1-\chi_{j}\right) I,
$$

где $b_{0 j}^{ \pm}(x)=\chi_{j}\left(a_{0}^{ \pm}(x)\right)^{-1} a_{0}^{ \pm}(x), b_{j}^{ \pm}(x)=b_{0 j}^{ \pm}(x)+1-\chi_{j}$.

Ясно, что $b_{j}^{ \pm}(x)=a^{ \pm}(x)$, когда $x \in \Gamma_{j}^{\prime \prime}$, и $b_{j}^{ \pm}(x)=1$, когда $x \in \Gamma \backslash \Gamma_{j}^{\prime}$, $j=1, \ldots, L$. Нетрудно убедиться, что

$$
A=A_{0} A_{1} \ldots A_{N}+T, \quad \text { где } \quad T \in \mathfrak{K}\left(L_{p}(\Gamma, \omega)\right) .
$$

Таким образом, из формулы (4.3) следует, что

$$
\text { Ind } A=\sum_{j=0}^{N} \operatorname{Ind} A_{j} \text {. }
$$

Нетрудно видеть, что

$$
\text { Ind } A_{0}=-\sum_{j=1}^{L}(2 \pi)^{-1}\left[\arg a_{+}(x) a_{-}^{-1}(x)\right]_{\gamma_{j\left(\zeta_{j}^{\prime}, \zeta_{j}^{\prime \prime}\right)}} .
$$

В формуле (4.4) точки $\zeta_{j}^{\prime}, \zeta_{j}^{\prime \prime}$ произвольны и лежат в области, где $a_{ \pm}(x)=1$.

Рассмотрим теперь вопрос об индексе оператора $A_{j}$. Оператор $A_{j}$, очевидно, фредгольмов в $L_{p}(\Gamma, \omega)$ тогда и только тогда, когда фредгольмов оператор $A_{j}^{\prime}=$ 
$\left.A_{j}\right|_{L_{p}\left(\Gamma_{j}^{\prime},\left|x-x_{j}\right|^{\beta_{j}}\right)}$, так как ограничение $\left.A_{j}\right|_{L_{p}\left(\Gamma \backslash \Gamma_{j}^{\prime},\left|x-x_{j}\right|^{\beta_{j}}\right)}=I$ есть единичньй оператор. При этом

$$
\text { Ind } A_{j}=\operatorname{Ind} A_{j}^{\prime} \text {. }
$$

Пространство $L_{p}\left(\Gamma_{j}^{\prime},\left|x-x_{j}\right|^{\beta_{j}}\right)$ реализуем как прямую сумму:

$$
L_{p}\left(\Gamma_{j}^{\prime},\left|x-x_{j}\right|^{\beta_{j}}\right)=L_{p}\left(\gamma_{j}^{+},\left|x-x_{j}\right|^{\beta_{j}}\right) \oplus L_{p}\left(\gamma_{j}^{-},\left|x-x_{j}\right|^{\beta_{j}}\right),
$$

где $\gamma_{j}^{+}$и $\gamma_{j}^{-}-$правая и левая части дуги $\Gamma_{j}^{\prime}$. В соответствии с таким представлением оператор $A_{j}^{\prime}$ реализуется как матричный оператор в $L_{p}\left(\gamma_{j}^{+},\left|x-x_{j}\right|^{\beta_{j}}\right) \oplus$ $L_{p}\left(\gamma_{j}^{-},\left|x-x_{j}\right|^{\beta_{j}}\right)$. После замены переменных, распрямляющей дуги $\gamma_{j}^{ \pm}$, оператор $A_{j}^{\prime}$ переходит в оператор $\hat{A}_{j}: L_{p, \beta_{j}}^{2}\left(\left(0, h_{j}\right)\right) \rightarrow L_{p, \beta_{j}}^{2}\left(\left(0, h_{j}\right)\right)$. Оператор $\hat{A}_{j}$ является сужением на $L_{p, \beta_{j}}^{2}\left(\left(0, h_{j}\right)\right)$ оператора $\mathscr{A}_{j} \in \mathfrak{D}^{2}\left(p, \beta_{j}, \delta_{j}\right)$ с символом, равным $\sigma_{x_{j}}(A)(t, \lambda)$, когда $(t, \lambda) \in\left(0, h_{j}^{\prime}\right) \times \mathbb{R}, h_{j}^{\prime}<h_{j}$, и равным 1 на множестве $\left[h_{j},+\infty\right) \times \mathbb{R}$. Согласно предложению 2.2 Ind $\hat{A}_{j}=$ Ind $\mathscr{A}_{j}$.

Вычисляя Ind $\mathscr{A}_{j}$ по формуле (2.8), получаем равенство

$$
\begin{aligned}
\text { Ind } \mathscr{A}_{j}= & -(2 \pi)^{-1}\left[\arg \operatorname{det} \sigma_{x_{j}}(A)\left(t_{j}, \lambda\right)\right]_{\lambda=-\infty}^{\infty} \\
& -(2 \pi)^{-1}\left[\arg \operatorname{det} \sigma_{x_{j}}(A)(t,+\infty) \sigma_{x_{j}}^{-1}(A)(t,-\infty)\right]_{t=t_{j}}^{h_{j}} .
\end{aligned}
$$

Положим теперь в формуле (4.4) $\zeta_{j}^{\prime}=x_{j}+\varphi_{j}^{+}\left(h_{j}\right), \zeta_{j}^{\prime \prime}=x_{j+1}+\varphi_{j+1}^{-}\left(h_{j}\right)$. Так каK

$$
\sigma_{x_{j}}(A)(t, \pm \infty)=\left|\begin{array}{cc}
a_{ \pm}\left(x_{j}+\varphi_{j}^{+}(t)\right) & 0 \\
0 & a_{ \pm}\left(x_{j}+\varphi_{j}^{-}(t)\right)
\end{array}\right|,
$$

то из формул (4.4), (4.5) следует формула индекса (4.2).

ЗАмЕЧАнИЕ. Формула индекса, аналогичная формуле (4.2), справедлива и для оператора (4.1) на сложном незамкнутом контуре с узлами, являющимися точками завихрения.

Аналогичная формула индекса справедлива и для операторов из банаховой алгебры $\mathfrak{M}_{p}(\Gamma, \omega)$.

\section{Список литературы}

1. Gohberg I. C., Krupnik N. J. Einfuhrung in die Theorie der eindimentionalen Singullaren Integral Operatoren. Basel: Birkhauser, 1979.

2. Krupnik N. Banach algebras with symbol and singular integral operators. Basel: Birkhauser, 1987.

3. Roch S., Silberman B. Algebras of convolution operators and their image in the Calkin algebras. Report Math. 90-05. Karl-Weierstrass-Ins. Math. Berlin: Akad. Wiss. DDr, 1990.

4. Bottcher A., Silberman B. Analysis of Toeplitz operators. Berlin: Springer-Verlag, 1990.

5. Симоненко И. Б., Чинь Нгок Минь. Локальный принцип в теории одномерных сингулярных уравнений с кусочно-непрерывными коэффициентами. Ростов-на-Дону: Рост. госуд. ун-т, 1986.

6. Roch S., Silberman B. The structure of algebras of singular integral operators // J. Integral Equat. and Appl. 1992. V. 4. № 3. P. 421-442.

7. Пламеневский Б. А., Сеничкин В. Н. О $C^{*}$-алгебрах сингулярных интегральных операторов с разрывными коэффициентами на сложном контуре // Изв. ВУЗов. Матем. 1984. №1. C. $25-33 ;$ 1984. № 4. P. 37-46. 
8. Gohberg I., Krupnik N., Spitkovskiy I. Banach algebras of singular integral operators with piecewise continuous coefficients. General contour and weight // J. Integral. Equat. and Appl. 1993. V. 17. P. 322-337.

9. Дудучава P. В., Лоцабидзе Т., Сагинашвили А. Сингулярные интегральные операторы на кривых с каспами // Сообщения АН ГрузССР. 1992. Т. 146. № 1. С. 21-24.

10. Шапиро М. В. Алгебра сингулярных операторов с карлемановским сдвигом на составном контуре // Сиб. матем. журн. 1978. Т. 19. № 1. С. 201-216.

11. Power S. C. Hankel operators with PQC symbols and singular integral operators // Proc. London. Math. Soc. 1980. V. 41. P. 45-65.

12. Sarason D. Toeplitz operator with piecewise quasicontinuous symbols // Indiana Univ. Math. J. 1977. V. 26. № 5. P. 817-838.

13. Bottcher A., Spitkovskiy T. Toeplitz Operators with PQC symbols and weighted Hardy spaces // J. Funct. Anal. 1991. V. 97. № 1. P. 194-214.

14. Silberman $B$. The $C^{*}$-algebra generated by Toeplitz and Hankel operators with piecewise quasicontinuous symbols // Integral Equation and Operator Theory. 1987. V. 10. P. $730-738$.

15. Суйфулаев Р. К. Краевая задача Римана на негладких открытых кривых // Матем. сб. 1980. T. 112. C. $147-161$.

16. Bottcher A., Karlovich Yu. I. Toeplitz and singular integral operators on Carleson curves with logarithmic whirl points // Integral Equations and Operator Theory. 1995. V. 22. P. 127-161.

17. Рабинович B. С. Псевдодифференциальные операторы Меллина и сингулярные интегральные операторы на сложных контурах с осциллирующей касательной // ДАН СССР. 1991. T. 321. № 5. C. $692-696$.

18. Федосов Б. В. Аналитические формулы индекса эллиптических операторов // Тр. моск. матем. об-ва. 1974. Т. 30. С. 159-241.

19. Хермандер Л. Анализ линейных дифференциальных операторов с частными производными. Т. 3. Псевдодифференциальные операторы. М.: Мир, 1987.

20. Taylor M. E. Pseudodifferential Operators. Princeton, New Jersey: PUP, 1981.

21. Грушин В. В. Псевдодифференциальные операторы на $\mathbb{R}^{n}$ с ограниченными символами // Функц. анализ и его прилож. 1970. № 4. С. 202-212.

22. Рабинович В. С. Нётеровость псевдодифференциальных операторов с символами класса $S_{\rho, \delta}^{m}(0 \leqslant \delta=\rho<1) / /$ Матем. заметки. 1980. Т. 27. С. 226-231.

23. Beals $R$. Characteriszation of pseudo-differential operators and applications // Duke Math. J. 1977. V. 44. P. 45-57.

Ростовский государственный университет

Поступило в редакцию 